\title{
Fast beam-based alignment using ac excitations
}

\author{
Zeus Martí@," Gabriele Benedetti, and Ubaldo Iriso \\ ALBA Synchrotron, C. de la Llum 2-26, 08290 Cerdanyola del Vallès, Spain \\ Andrea Franchiø \\ ESRF, CS 40220, 38043 Grenoble CEDEX 9, France
}

(Received 1 October 2019; published 22 January 2020)

\begin{abstract}
Standard quadrupole beam-based alignment (BBA) techniques rely on orbit data and on the sequential variation of quadrupole and orbit corrector magnets (OCM). This results in time-consuming measurements of the order of several hours in most circular accelerators. Fast $(10 \mathrm{kHz})$ beam position monitors (BPM) and OCMs with ac power supplies are routinely used in modern synchrotron light sources to drive fast orbit feedback systems. In this paper we show how they can be employed also to dramatically reduce the time for any quadrupole BBA to several minutes only, ensuring the same level of accuracy and precision. Moreover, conversely to the standard BBA, the new procedure accounts automatically for any level of betatron coupling, BPM roll and OCM tilt. In the case of the ALBA 3rd generation light source, the time for a complete measurement dropped from 5 hours to 10 minutes, a reduction by a factor 30 . As further extension of this novel approach, an even faster skew quadrupole BBA was demonstrated in ALBA for the first time, taking advantage of the additional ac modulation of the skew quadrupole field. Results from this fully ac measurement are compared with those obtained via a traditional dc scan of the skew quadrupole.
\end{abstract}

DOI: 10.1103/PhysRevAccelBeams.23.012802

\section{INTRODUCTION}

Aligning the beam centroid to the center of the magnetic elements is an efficient way to minimize unwanted multipole feed-down effects in particle accelerators. A beam crossing magnets off axis experiences orbit distortion and alteration of the dispersion function (in quadrupoles via dipole feeddown), as well as linear optics errors and coupling (in sextupoles via normal and skew quadrupole feed-down). The beam-based alignment (BBA) facilitates the work of corrector magnets to minimize these errors and reduces the deviation between the real machine and the accelerator model. Two different quadrupole BBA approaches are described in the literature. In the first, the observable is the offset between beam trajectory and quadrupole axis [1-4]. In the second, the observable is the beam position at the BPM when it passes through the axis of the closest quadrupole [5-8]. The first technique is known as beam-toquad while the second is referred to as BPM-to-quad.

The beam-to-quad technique does not require OCM scans but is model dependent: Tunes and optical functions are needed to infer the beam-to-quad offset. Moreover, it

\footnotetext{
*zeus@cells.es

Published by the American Physical Society under the terms of the Creative Commons Attribution 4.0 International license. Further distribution of this work must maintain attribution to the author(s) and the published article's title, journal citation, and DOI.
}

does not provide directly the BPM-to-quad offsets, though they can be interpolated from the measured ones. This method gives a direct indication of how well centered is the beam, but not how much the individual quadrupoles or the BPMs have to be realigned. To achieve that the model has to be used again.

The BPM-to-quad technique does not rely on the accelerator optics model but needs to scan the strength of one or several OCMs at every quadrupole change. This is usually much more time consuming and it may give inaccurate results if the beam orbit has a pronounced angle at the quadrupole. The aim of the method is to align the BPMs, which are prone to electronic induced offsets, to the magnets layout, which is assumed to be more stable over time. Besides, the BPMs are usually attached to the vacuum chamber and need to be realigned after interventions. Magnets are misaligned too, but this lays outside the scope of this method. In synchrotron light sources the quadrupole scan is typically performed step by step in a dc way and slow acquisition $(10 \mathrm{~Hz})$ orbit position data are analyzed, whereas the BBA of quadrupoles in the interaction regions of colliders is usually carried out via a continuous quadrupole gradient modulation and the harmonic analysis of turnby-turn BPM data. In the literature the latter approach is referred to as k-modulation [5] and requires quadrupole power supplies with ac capabilities, presently not available in ALBA. Therefore, standard BPM-to-quad BBA used to be carried out in its storage ring via slow acquisition orbit position data and dc changes of both quadrupoles and 
OCMs [9]. A complete measurement over 120 BPMs in both planes takes usually 5 hours. An overview of different BBA techniques and implementations can be found in [10].

In this paper we present a fast BBA (FBBA) BPM-to-quad method based on the parallel ac modulation of several OCMs at different frequencies and on the harmonic analysis of orbit data sampled at high frequency $(10 \mathrm{kHz})$. A complete FBBA requires 10 minutes only, which represents a reduction by a factor 30 compared to the standard 5 hours. It is worthwhile stressing the fact that the FBBA is a different scheme compared to the k-modulation, where the ac modulation is performed on the quadrupoles, not on the steerers, and the harmonic analysis is performed on the beam trajectory (turn-by-turn data), not on the orbit.

A fully ac FBBA with harmonic modulation of the magnet to be centered was proved for the first time at ALBA by centering the beam into skew quadrupoles, which are trim coils mounted on the main sextupoles and feature ac capabilities. Besides the fact of being an even faster operation, this analysis aligns automatically also the sextupole magnets onto which the skew quadrupole coils are installed, with benefits to the machine optics.

A fast acquisition archiver (FA) providing synchronous BPM data at $10 \mathrm{kHz}$ rate originally developed at Diamond [11] was installed in ALBA [12] and other synchrotron light sources since 2011. OCMs can be then excited in parallel in both planes and at different frequencies. The motion induced by any steerer in each plane can be isolated and analyzed separately from the harmonic analysis of FA data during the OCM modulation by looking at the spectral peaks generated at those (known) frequencies. Even though FA data contain position data synchronized among all $120 \mathrm{BPMs}$, this is not the case for the ac OCMs. Their currents are either not archived (as in the case of ALBA) or not suitable for conversion into strengths, also their calibration factors are frequency-dependent and not known a priori. This requires some preliminary mathematical gymnastics of the original BBA formulas to remove any dependence of the observables on the OCM strength. The formalism developed to this end accounts also for the presence of an arbitrarily large betatron coupling, OCM tilt, and BPM roll.

The paper is structured as follows: the FBBA is presented in Sec. II. In Sec. III the experimental set-up is described. The error analysis based on the alignment tolerances and the expected accuracy are evaluated in Sec. IV. In Sec. V experimental results of the new methodology applied to ALBA are presented and compared with the standard BBA. Some conclusions are drawn in Sec. VI, whereas the mathematical details of the proposed approach are presented in Appendices A and B.

\section{QUADRUPOLE FAST BEAM-BASED ALIGNMENT}

In this section we present different approaches to evaluate the BPM-to-quad offsets with respect to quadrupole magnets under various assumptions and conditions. In Sec. II A we consider exciting horizontal and vertical OCMs separately while in Sec. II B we consider a simultaneous orbit modulation in the two planes, though still ignoring coupling effects. A complete treatment including betatron coupling, BPM roll, OCM tilt and dual-plane orbit modulation is presented in Sec. II C. While the first two approaches are valid for normal quadrupoles only, the last is more general and is applicable to both normal and skew quadrupoles. A further extension to make use of an ac modulation of the quadrupole itself (either normal or skew) on top of the ac modulation of the OCMs is also presented. If implemented, this fully ac variant would further reduce the FBBA time by an additional factor two.

\section{A. Single-plane ac excitation}

In the linear regime and in the presence of a time-varying OCM setting, $v_{j}(t)$, the vertical closed-orbit readings $y_{l}(t)$ and $y_{k}(t)$ at two BPMs $l$ and $k$ read

$$
\begin{aligned}
& y_{l}(t)-y_{l}^{0}=R_{\mathrm{lj}}^{\mathrm{yy}}\left(v_{j}(t)-v_{0}\right), \\
& y_{k}(t)-y_{k}^{0}=R_{\mathrm{kj}}^{\mathrm{yy}}\left(v_{j}(t)-v_{0}\right),
\end{aligned}
$$

where $t$ represents discrete, equally spaced time values (sampled at a $10 \mathrm{kHz}$ rate in the case of ALBA), $y^{0}$ is the $\mathrm{BPM}$ position reading when the OCM setting is $v_{0}$. As any other BPM-to-quad BBA implementation, this method does not relies on the accelerator model knowledge so the effective orbit response matrix (ORM) $R_{\mathrm{lj}}^{\mathrm{yy}}$ is unknown. In this paper we will assume that the BPM $l$ is next to the varied quadrupole as sketched in Fig. 1. The OCM $j$ is chosen to produce a large variation of the orbit at BPM $l$. Hence, although we do not assume any specific value for $R_{\mathrm{lj}}^{\mathrm{yy}}$, it should not have a small value at BPM $l$.

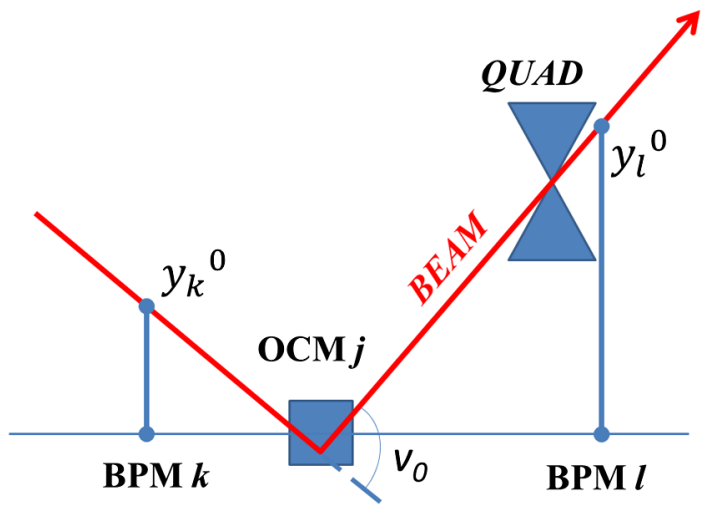

FIG. 1. Sketch of the beam closed orbit when passing through the center of the quadrupole (next to the BPM $l$ ) to be aligned. In the example drawn $v_{j}(t)=v_{0}$ generates the closed orbit that makes the beam pass through the quadrupole center. 
While a synchronous temporal sampling of the BPM data is ensured by the FA, in the case of ALBA, the OCMs data $v_{j}(t)$ are not available, hence not observable. Nevertheless, the two equations in the above system can be combined to remove any dependence on the OCM excitation, namely

$$
y_{l 1}(t)-y_{l 1}^{0}=\frac{R_{\mathrm{lj} 1}^{\mathrm{yy}}}{R_{\mathrm{kj} 1}^{\mathrm{yy}}}\left(y_{k 1}(t)-y_{k 1}^{0}\right) .
$$

The above equation describes the linear correlation between BPM data during a beam steering. The label 1 refers to the first quadrupole setting. Any change in the quadrupole current $\Delta I$ modifies both the response matrix elements, i.e., the slope in Eq. (2), and the orbit distortion if the beam is not centered to the quadrupole under study. These effects can be written as

$$
y_{l 2}(t)-y_{l 2}^{0}=\frac{R_{\mathrm{lj} 2}^{\mathrm{yy}}}{R_{\mathrm{kj} 2}^{\mathrm{yy}}}\left(y_{k 2}(t)-y_{k 2}^{0}\right),
$$

where the label 2 refers to the modified quadrupole strength (or modified quadrupole current, $I_{0}+\Delta I$ ). The new constant values can be written as $y_{l 2}^{0}=y_{l 1}^{0}+A_{l}$ and $y_{k 2}^{0}=y_{k 1}^{0}+A_{k}$, where $A_{l}$ and $A_{k}$ account for the orbit distorted by the off-axis passage through the quadrupole. Equation (3) can be then rewritten as

$$
y_{l 2}(t)-y_{l 1}^{0}=\frac{R_{\mathrm{lj} 2}^{\mathrm{yy}}}{R_{\mathrm{kj} 2}^{\mathrm{yy}}}\left(y_{k 2}(t)-y_{k 1}^{0}\right)+A_{l k j},
$$

where $A_{l k j}=A_{l}-R_{\mathrm{lj} 2}^{\mathrm{yy}} / R_{\mathrm{kj} 2}^{\mathrm{yy}} A_{k}$ represents an offset with respect to the line of Eq. (2). The intersection between two (or more) lines obtained with two (or more) quadrupole settings would correspond to the beam crossing the quadrupole on axis, since the orbit distortion would not depend on its strength. According to Eqs. (2) and (4), the only condition for two lines to have the same value is when both hand sides are zero, i.e., when $A_{l k j}=0 \Rightarrow y_{l 2}^{0}=y_{l 1}^{0}$, $y_{k 2}^{0}=y_{k 1}^{0}, y_{l}(t)=y_{l 1}^{0}$ and $y_{k}(t)=y_{k 1}^{0}$. The physical meaning of $y_{l 1}^{0}$ and $y_{k 1}^{0}$ in Eq. (2) is then nothing else than the BPM readings for which the beam crosses the quadrupole on axis, i.e., when the beam is aligned. If one of the monitors is close to the quadrupole under scrutiny, e.g., the BPM $l, y_{l}^{0}$ denotes the BPM-to-quad offset. The offset can be obtained not knowing the exact value of the response matrix $R_{\mathrm{lj}}^{\mathrm{yy}}$, which depends on the OCM and BPM calibration factors. This technique, as well as the original BBA, is completely model independent.

A graphical illustration of the method is given in Fig. 2 where the two lines measured prior and after a change in the quadrupole current, corresponding to Eqs. (2) and (3), are displayed. In this particular case, using only

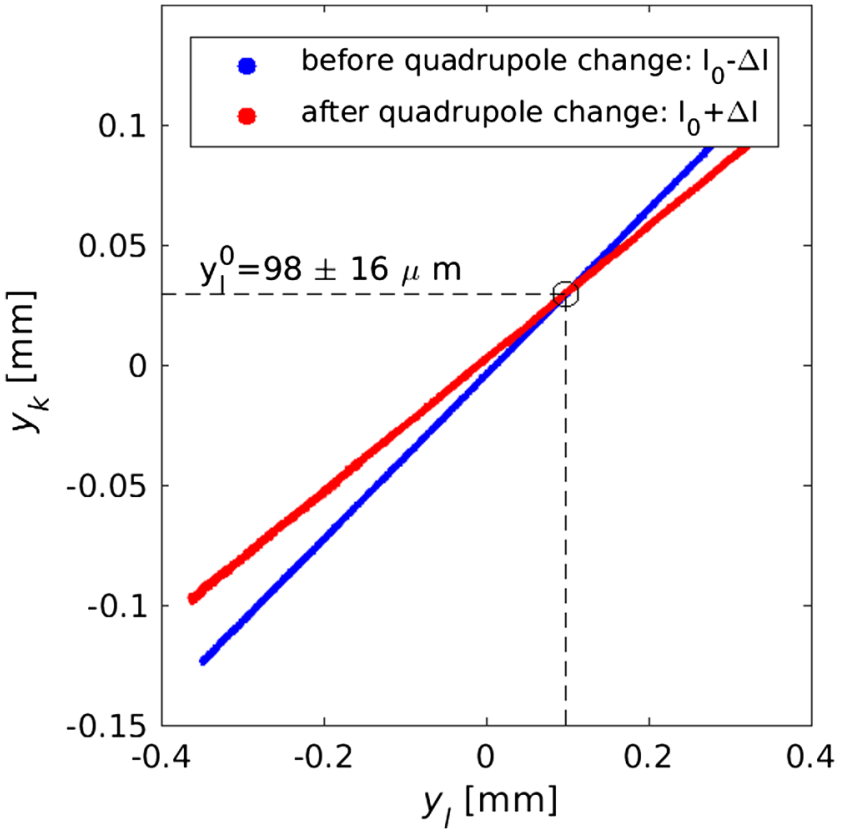

FIG. 2. Example of measured only vertical plane FBBA. The BPM offsets leading to the on-axis crossing of the beam through the quadrupole are inferred from the intercept of the two lines: before (blue) and after (red) the quadrupole change $\Delta I=2.5 \mathrm{~A}$. The OCM was excited at $6 \mathrm{~Hz}$. The data shown here corresponds to the case $l=69$.

the information of one BPM (BPM $k$ ) apart from the BPM closest to the quadrupole (BPM $l$ ), the estimated offset is $98 \pm 16 \mu \mathrm{m}$. The error bar is computed as the distance along the axis of the BPM $l$ from the intersection point when a separation between the two fit lines equals the BPM noise. The latter is evaluated from the fluctuations in the BPM readings with unperturbed beam.

Accuracy and precision can be improved by using data from several BPMs $k$. Ideally, the two lines of Fig. 2 shall have sufficiently different slopes so to increase both the accuracy, when determining the intersection, and the precision, when computing the error bar. Similarly, different pairs of BPMs provide different accuracy and precision, because of their different sensitivity to determine the offset, that is proportional to the change of the slopes in Eqs. (2) and (3), namely $\frac{R_{\mathrm{l} j}^{\mathrm{yy}}}{R_{\mathrm{kj} 2}^{y y}}-\frac{R_{\mathrm{j} 1}^{\mathrm{yy}}}{R_{\mathrm{kj} 1}^{\mathrm{yy}} \text {. }}$

For each quadrupole, the results presented here have been obtained first by selecting the most suitable OCM and then by averaging the results over different pairs of BPMs. Couples with error bars larger than three times the average error bar (i.e., with weak correlation) are discarded. In the example of Fig. 2, by using the data from all the BPMs a simple average leads to an offset of $84 \pm 10 \mu \mathrm{m}$. Instead, if the above mentioned outliers are discarded, the final offset is $92 \pm 3 \mu \mathrm{m}$. Figure 3 shows the offset estimation of BPM No. 69 given by each one of the other 119 BPMs. The red 1 dots represent the discarded BPM results and correspond 


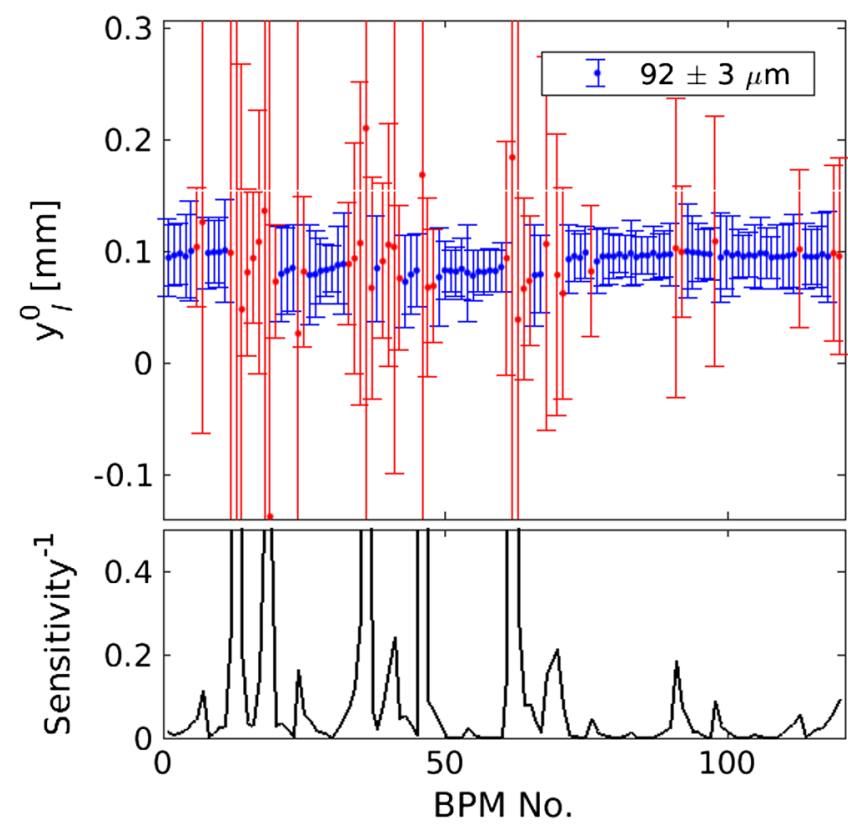

FIG. 3. The upper plot shows the BPM $l$ estimated offsets obtained from the lines intersection using data from all the other 119 BPMs. The cases where the error bar is larger than three times the average error bar are highlighted in red and removed from the final average. The lower plot shows the inverse of the difference between the slopes in Eqs. (2) and (3) estimated from the model. Notice that the removed BPMs (red dots) in the upper plot coincide with those for which the such sensitivity factor is close to zero (the inverse factor has a peak) in the lower plot. The data shown here corresponds to the case BPM $l=69$.

well with peaks of the inverse of the sensitivity factor estimated from the model.

It is generally assumed that the quadrupoles are better aligned and keep their alignment over time better than the BPMs. In most cases, after each measurement, an artificial BPM numerical offset $y_{l 1}^{0}$ is added to ensure that the closed-orbit correction centers the beam onto the quadrupole axis.

\section{B. Multi-frequency and dual-plane ac excitation}

The analysis presented in the previous section does not depend on the frequency of the ac OCM excitation. In order to fully exploit the ac nature of this approach and to speed up the overall measurement, quadrupole offset in both planes can be obtained in a single measurement by a simultaneous excitation of horizontal and vertical OCMs at different frequencies. The FA data stream will contain the beam response to both excitations, and so a Fourier analysis can be performed to decouple the two responses. In Eq. (2) the $y_{l}(t)$ signal contains the orbit response both from the dc OCM strength and the (not observable) ac OCM excitation $v_{j}(t)$. When the beam is excited by two OCMs (horizontal and vertical) at different frequencies, both $\mathrm{dc}$ and ac originated components need to be isolated and summed up before applying the method described in the previous section. Equation (2) is generalized to

$$
\begin{aligned}
& \tilde{x}_{l}(t)-x_{l}^{0}=\frac{R_{\mathrm{lj}}^{\mathrm{xx}}}{R_{\mathrm{kj}}^{\mathrm{xx}}}\left(\tilde{x}_{k}(t)-x_{k}^{0}\right), \\
& \tilde{y}_{l}(t)-y_{l}^{0}=\frac{R_{\mathrm{lj}}^{\mathrm{yy}}}{R_{\mathrm{kj}}^{\mathrm{yy}}}\left(\tilde{y}_{k}(t)-y_{k}^{0}\right),
\end{aligned}
$$

where

$$
\begin{aligned}
& \tilde{x}_{l}(t)=x_{l, d c}+x_{l, f_{h}}(t), \\
& \tilde{y}_{l}(t)=y_{l, d c}+y_{l, f_{v}}(t) .
\end{aligned}
$$

$x_{d c}$ and $y_{d c}$ denote the dc originated components of the FA data, whereas $x_{f_{h}}(t)$ and $y_{f_{v}}(t)$ represent their harmonics oscillating at the horizontal and vertical excitation frequencies, $f_{h}$ and $f_{v}$, respectively. Any BPM response due to other external sources is hence removed. Under the assumption of operating the machine at low betatron coupling, its impact can be ignored and the analysis of Sec. II A repeated on the signals $\tilde{x}$ and $\tilde{y}$.

Before showing an example of the multifrequency FBBA, it shall be noticed that betatron coupling and the finite sampling frequency $(10 \mathrm{kHz})$ introduce some crosstalk among the BPM harmonics. Even in machines operating at low coupling, this interference may appear at those BPMs with weak response to OCM excitation. This issue is addressed in Appendix B, where a numerical solution is presented to correct the cross-talk. The cross talk depends on how close are the selected ac frequencies of each plane but there are other considerations that influence the frequency selection and are addressed in Sec. III. In the case of ALBA the most suitable frequencies were found to be $7 \mathrm{~Hz}$ in the horizontal plane and $6 \mathrm{~Hz}$ in the vertical plane.

As for the single-plane, single-frequency measurement introduced in the previous section, the intersection of the lines of Eq. (5) before and after the quadrupole change provides the BPM-to-quad offsets, $x_{l}^{0}$ and $x_{k}^{0}$. The same exercise applied to the vertical data via Eq. (6) will determine the vertical BPM-to-quad offsets.

In Fig. 4 results from a (parallel) dual-plane excitation are displayed along with those from the equivalent (sequential) single-plane excitations. In the top plot the raw vertical data sampled at $10 \mathrm{kHz}$ (corresponding to Eq. (2) are reported. The blue and red lines refer to the single-plane excitation. The green and black curves are from the dualplane excitation, whose beating is generated by betatron coupling mixing the two modulations. The $\tilde{y}$ data of Eq. (6) obtained from the harmonic analysis of those curves are reported in the bottom plot, showing how the two lines obtained from the dual-frequency OCM excitation match 

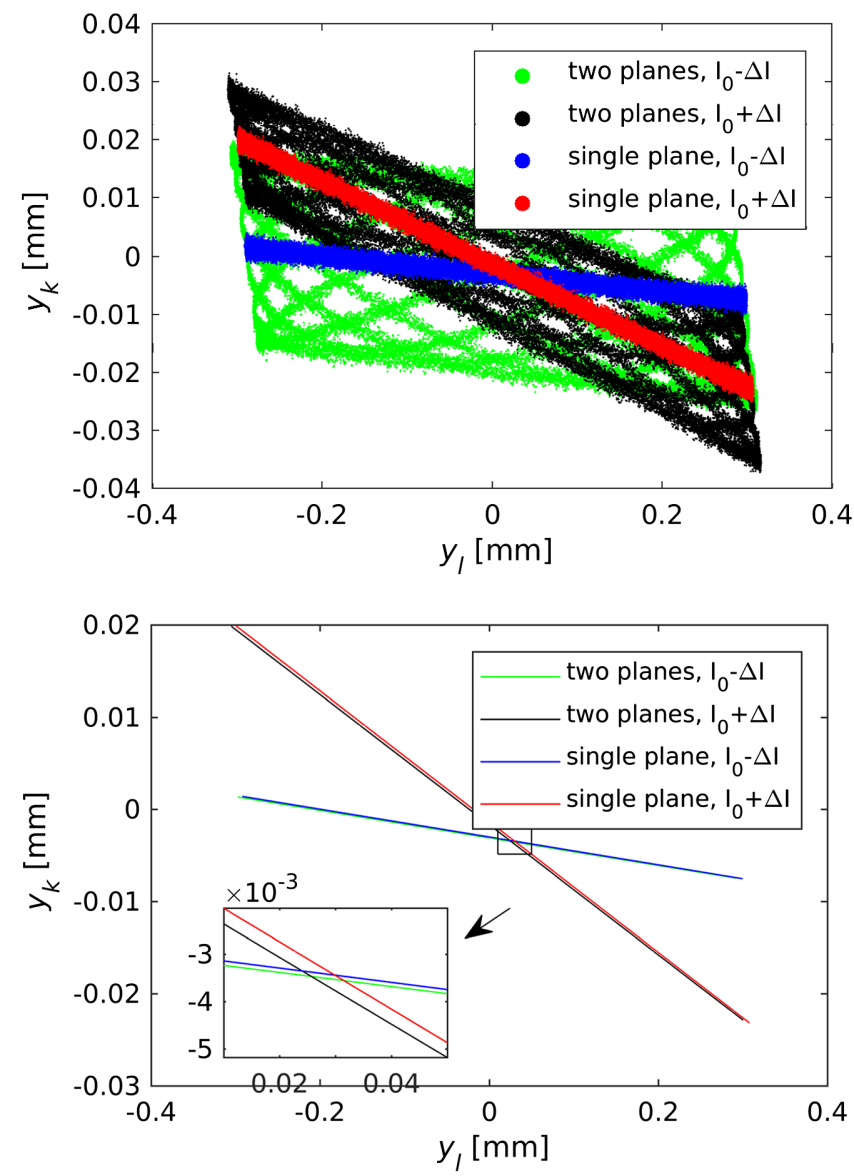

FIG. 4. Top plot: correlation between two BPMs vertical raw data sampled at $10 \mathrm{kHz}$ before (green dots) and after (black dots) a quadrupole change during the simultaneous excitation of a horizontal (at $7 \mathrm{~Hz}$ ) and a vertical (at $6 \mathrm{~Hz}$ ) OCM. Blue and red dots correspond to data taken with one OCM at a time. Bottom plot: comparison between the lines resulting from fitting the single-frequency case with Eq. (2) and those of Eqs. (6) and (8) from the dual-frequency case. The two final offsets agree roughly: $32 \pm 4 \mu \mathrm{m}$ from the single-frequency excitation and $29 \pm 2 \mu \mathrm{m}$ from the dual-plane modulation. The data shown here corresponds to the case BPM $l=5$.

very well the linear fits obtained from the single-frequency modulation. In this example, the offsets of the closest BPM to the quadrupole obtained from the two approaches are $32 \pm 4 \mu \mathrm{m}$ (single frequency) and $29 \pm 2 \mu \mathrm{m}$ (dual frequency). These results are obtained after applying the same statistics described at the end of Sec. II A.

\section{Dual-plane ac excitation and beam coupling}

If betatron coupling, BPMs rolls or OCMs tilts cannot be neglected, Eq. (1) needs to be modified by including the effective off-diagonal ORM terms $R^{\mathrm{xy}}, R^{\mathrm{yx}}$

$$
\begin{aligned}
& x_{k}(t)-x_{k}^{0}=R_{\mathrm{kj}}^{\mathrm{xx}}\left(h_{j}(t)-h_{0}\right)+R_{\mathrm{kp}}^{\mathrm{xy}}\left(v_{p}(t)-v_{0}\right) \\
& y_{n}(t)-y_{n}^{0}=R_{\mathrm{np}}^{\mathrm{yy}}\left(v_{p}(t)-v_{0}\right)+R_{\mathrm{nj}}^{\mathrm{yx}}\left(h_{j}(t)-h_{0}\right) .
\end{aligned}
$$

By doing so, the ratio between the above two equations evaluated at two BPMs does not yield any longer the observable linear relation of Eq. (2). A different (and novel) mathematical approach is then required.

Equation (9) is general enough to be valid for any amount of OCM tilts. The inclusion of $R^{\mathrm{xy}}$ and $R^{\mathrm{yx}}$ can absorb coupling effects of any kind. A brief proof is presented in Appendix Sec. A 1 of Appendix A.

The inclusion of betatron coupling allows a straightforward extension of the FBBA to skew quadrupoles. This is of particular interest in synchrotron light sources since these magnets are usually trim coils mounted on the yokes of sextupoles: aligning the beam at a skew quadrupole would hence correspond to a sextupole BBA, which benefits the machine linear optics and betatron coupling. Moreover, since trim coils usually feature ac functionalities, the FBBA can be extended to a fully ac approach by varying their strengths sinusoidally instead of repeating the measurement at two different strengths, hence halving the measurement time. The frequency of the skew quadrupole excitation is chosen so to not interfere with the ones of the OCMs.

In this sections only the final results are presented and discussed, while all proofs and details can be found in Appendix A. There, a general formula evaluating the BPMto-quad offset $l$ is derived:

$$
\begin{aligned}
& x_{l}^{0}=\Re\left\{\left\langle x_{l} \mid 0\right\rangle\right\}+\mathcal{S}\left\{\left\langle x_{l} \mid f_{h}\right\rangle\right\} \mathcal{M}_{h}+\mathcal{S}\left\{\left\langle x_{l} \mid f_{v}\right\rangle\right\} \mathcal{M}_{v} \\
& y_{l}^{0}=\Re\left\{\left\langle y_{l} \mid 0\right\rangle\right\}+\mathcal{S}\left\{\left\langle y_{l} \mid f_{v}\right\rangle\right\} \mathcal{M}_{v}+\mathcal{S}\left\{\left\langle y_{l} \mid f_{h}\right\rangle\right\} \mathcal{M}_{h},
\end{aligned}
$$

where the coefficients $\mathcal{M}$ are observable independent on the type of quadrupole (be it normal or skew), though they differ if the latter is changed in either dc or ac mode. The symbol $\langle x \mid f\rangle$ denotes the Fourier component of the signal $x$ at the frequency $f$, i.e., the projection of $x$ on $f$. The signed amplitudes $\mathcal{S}$ are defined as:

$$
\mathcal{S}\left\{\left\langle x \mid f_{z}\right\rangle\right\}=\left|\left\langle x \mid f_{z}\right\rangle\right| \operatorname{sgn}\left\{\cos \left(\psi_{x}^{(z)}-\psi_{z}\right)\right\},
$$

where $\psi_{x}^{(z)}$ is the phase of the BPMs signal $x$ at the steerer frequency $f_{z}$ corresponding to the $z$ plane while $\psi_{z}$ is the phase of that steerer signal. All projections and phases can be obtained from the BPM readings of the FA. Explicit formulas are given in Appendix A. There it is also shown how the difference $\psi_{x}^{(z)}-\psi_{z}$ is either 0 or $\pi$. Thus, the sign of $\mathcal{S}$ is either positive or negative and shall correspond to the one of the ORM coefficients.

$\Re\left\{\left\langle x_{l} \mid 0\right\rangle\right\}$ represents thus the real part of the $\mathrm{dc}$ component of the horizontal beam orbit during the simultaneous horizontal and vertical ac OCM excitation. $\mathcal{S}\left\{\left\langle x_{l} \mid f_{h}\right\rangle\right\}$ is the signed amplitude of the horizontal position at frequency $f_{h}$ and is the leading term along with the dc component. The term $\mathcal{S}\left\{\left\langle x_{l} \mid f_{v}\right\rangle\right\}$ accounts for betatron coupling and is proportional to the horizontal beam response to the vertical OCM. Equivalent definitions and considerations apply to the vertical data. 
The formulas in Eq. (10) are the core of the FBBA and apply to any quadrupole type (normal or skew) and modulation ( $\mathrm{dc}$ or ac). The coefficients $\mathcal{M}$ read

$$
\begin{gathered}
\mathcal{M}_{h}=-\frac{\mathcal{D}_{x} \mathcal{D}_{\mathrm{yv}}-\mathcal{D}_{\mathrm{xv}} \mathcal{D}_{y}}{\mathcal{D}_{\mathrm{xh}} \mathcal{D}_{\mathrm{yv}}-\mathcal{D}_{\mathrm{xv}} \mathcal{D}_{\mathrm{yh}}}=\frac{\mathcal{Y}_{h k}}{\mathcal{X}_{h k}} \\
\mathcal{M}_{v}=-\frac{\mathcal{D}_{\mathrm{xh}} \mathcal{D}_{y}-\mathcal{D}_{x} \mathcal{D}_{\mathrm{yh}}}{\mathcal{D}_{\mathrm{xh}} \mathcal{D}_{\mathrm{yv}}-\mathcal{D}_{\mathrm{xv}} \mathcal{D}_{\mathrm{yh}}}=\frac{\mathcal{Y}_{v k}}{\mathcal{X}_{v k}} .
\end{gathered}
$$

For a dc (normal or skew) quadrupole scan, the coefficients $\mathcal{D}$ become

$$
\begin{aligned}
\mathcal{D}_{\mathrm{x}} & =\Re\left\{\left\langle x_{k 2} \mid 0\right\rangle\right\}-\Re\left\{\left\langle x_{k 1} \mid 0\right\rangle\right\} \\
\mathcal{D}_{\mathrm{y}} & =\Re\left\{\left\langle y_{k 2} \mid 0\right\rangle\right\}-\Re\left\{\left\langle y_{k 1} \mid 0\right\rangle\right\} \\
\mathcal{D}_{\mathrm{xh}} & =\mathcal{S}\left\{\left\langle x_{k 2} \mid f_{h}\right\rangle\right\}-\mathcal{S}\left\{\left\langle x_{k 1} \mid f_{h}\right\rangle\right\} \\
\mathcal{D}_{\mathrm{yv}} & =\mathcal{S}\left\{\left\langle y_{k 2} \mid f_{v}\right\rangle\right\}-\mathcal{S}\left\{\left\langle y_{k 1} \mid f_{v}\right\rangle\right\} \\
\mathcal{D}_{\mathrm{xv}} & =\mathcal{S}\left\{\left\langle x_{k 2} \mid f_{h}\right\rangle\right\}-\mathcal{S}\left\{\left\langle x_{k 1} \mid f_{h}\right\rangle\right\} \\
\mathcal{D}_{\mathrm{yh}} & =\mathcal{S}\left\{\left\langle y_{k 2} \mid f_{h}\right\rangle\right\}-\mathcal{S}\left\{\left\langle y_{k 1} \mid f_{h}\right\rangle\right\} .
\end{aligned}
$$

An analytic derivation is given in Sec. A 2 of Appendix A. Labels 1 and 2 refer to data acquired at the quadrupole current $I_{0}-\Delta I$ and at $I_{0}+\Delta I$, respectively.
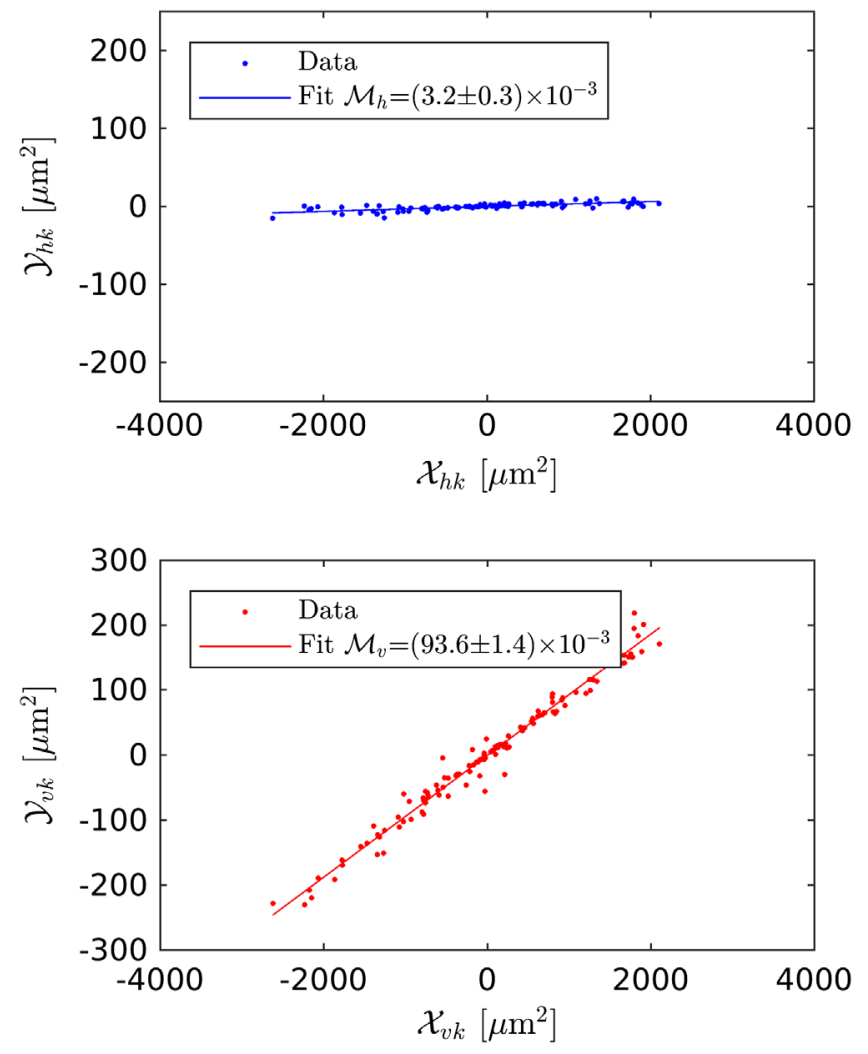

FIG. 5. Example of a $\mathcal{M}_{h}$ (top) and $\mathcal{M}_{v}$ (bottom) linear fit using the combined data of the 120 BPMs before and after a dc normal quadrupole changed by 2.5A. Quadrupole and steerer setting are the same of Fig. 4 i.e., BPM $l=5$. Using Eq. (10), the resulting vertical offset taking coupling effects into account is $y_{l}^{0}=30.5 \pm 0.4 \mu \mathrm{m}$.
In the case of ac excitation of the normal or skew quadrupole, the coefficients $\mathcal{D}$ read

$$
\begin{aligned}
\mathcal{D}_{\mathrm{x}} & =\mathcal{S}\left\{\left\langle x_{k} \mid f_{s}\right\rangle\right\} \\
\mathcal{D}_{\mathrm{y}} & =\mathcal{S}\left\{\left\langle y_{k} \mid f_{s}\right\rangle\right\} \\
\mathcal{D}_{\mathrm{xh}} & =\mathcal{S}\left\{\left\langle x_{k} \mid f_{h}+f_{s}\right\rangle\right\}+\mathcal{S}\left\{\left\langle x_{k} \mid f_{h}-f_{s}\right\rangle\right\} \\
\mathcal{D}_{\mathrm{yv}} & =\mathcal{S}\left\{\left\langle y_{k} \mid f_{v}+f_{s}\right\rangle\right\}+\mathcal{S}\left\{\left\langle y_{k} \mid f_{v}-f_{s}\right\rangle\right\} \\
\mathcal{D}_{\mathrm{xv}} & =\mathcal{S}\left\{\left\langle x_{k} \mid f_{v}+f_{s}\right\rangle\right\}+\mathcal{S}\left\{\left\langle x_{k} \mid f_{v}-f_{s}\right\rangle\right\} \\
\mathcal{D}_{\mathrm{yh}} & =\mathcal{S}\left\{\left\langle y_{k} \mid f_{h}+f_{s}\right\rangle\right\}+\mathcal{S}\left\{\left\langle y_{k} \mid f_{h}-f_{s}\right\rangle\right\} .
\end{aligned}
$$

An analytic derivation is given in Sec. A 3 of Appendix A. $f_{s}$ is the (known) skew quadrupole excitation frequency.

Throughout this paper, $\mathcal{M}_{h, v}$ are computed as the slopes of the linear fits $\mathcal{Y}_{h k}=\mathcal{M}_{h} \mathcal{X}_{h k}$ and $\mathcal{Y}_{v k}=\mathcal{M}_{v} \mathcal{X}_{v k}$, where the denominator $\mathcal{X}$ and numerator $\mathcal{Y}$ are measured at all BPMs. An example of such fits in the case of a normal dc varied quadrupole for BPM $l=5$ is shown in Fig. 5. The vertical offset measured via Eq. (10), i.e., taking betatron coupling into account, is $30.5 \pm 0.4 \mu \mathrm{m}$, compatible to the value obtained from uncoupled analysis of Fig. 4
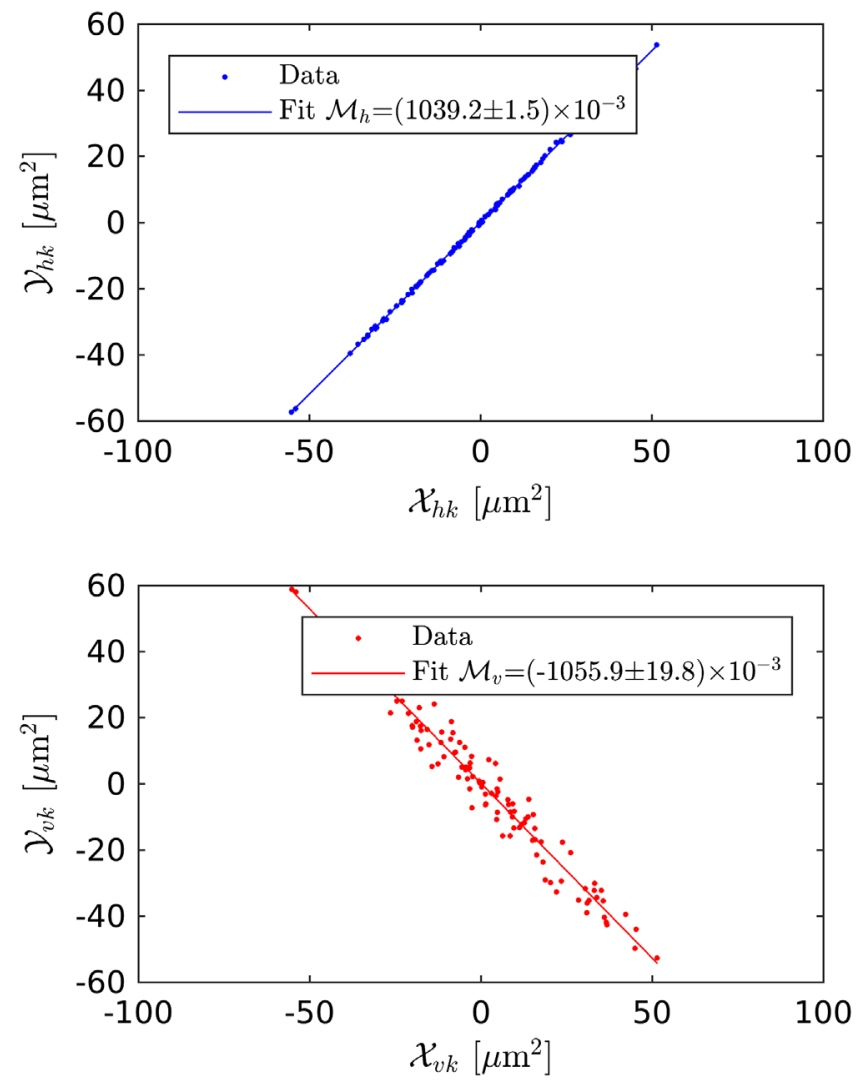

FIG. 6. Example of a $\mathcal{M}_{h}$ and $\mathcal{M}_{v}$ linear fit using the combined data of the 120 BPMs before and after a dc skew quadrupole changed by $2.5 \mathrm{~A}$. Using Eq. (10), the resulting offsets are $x_{l}^{0}=$ $330.7 \pm 0.5 \mu \mathrm{m}$ and $y_{l}^{0}=265.3 \pm 7.0 \mu \mathrm{m}$. The data shown here corresponds to the case $l=10$. 
$(29 \pm 2 \mu \mathrm{m})$ and the single-frequency excitation $(32 \pm 4 \mu \mathrm{m})$. For these specific quadrupole and BPM, the third (coupling) term in the r.h.s of Eq. (10) which is proportional to $\mathcal{M}_{h}$ is relatively small, $\simeq 0.1 \mu \mathrm{m}$. Larger deviations between this more general analysis and the one (ignoring coupling) described in the previous section are to be expected at quadrupoles with larger offsets (i.e., larger coefficients $\mathcal{M}$ ) and/or larger coupled motion (i.e., large cross-term signed amplitudes $\mathcal{S}\left\{\left\langle x_{l} \mid f_{v}\right\rangle\right\}$ and $\mathcal{S}\left\{\left\langle y_{l} \mid f_{h}\right\rangle\right\}$ ).

The uncertainty associated to the resulting BPM-to-quad offsets is obtained by error propagation in Eq. (10). The uncertainty associated to the $\mathcal{M}$ factors is obtained from the fit. The uncertainty associated to all projections $\langle x \mid f\rangle$ and $\langle y \mid f\rangle$ is obtained from the corresponding BPM data with neither OCM nor quadrupole excitation. An example of such baseline noise is shown in Fig. 9.

In order to test the validity of Eq. (9) for large BPM rolls, we reanalyzed the same data but rotating the $x$ and $y$ readings of all the BPMs by different angles, except BPM $l$. Irrespective to the angle artificially applied the result changes two orders of magnitude less that the assigned
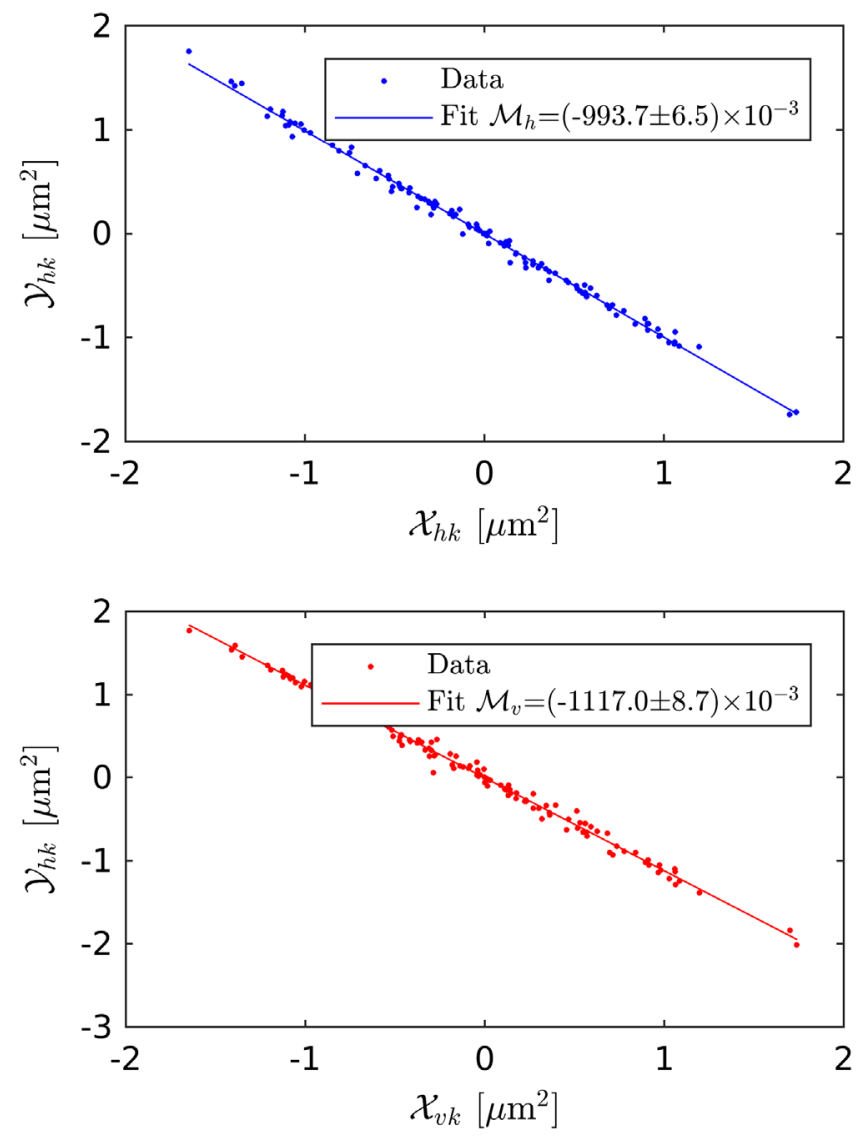

FIG. 7. Example of a $\mathcal{M}_{h}$ and $\mathcal{M}_{v}$ linear fit using the combined data of the 120 BPMs with a skew quadrupole ac modulation (at $1.6 \mathrm{~Hz}$ ) of the same skew quadrupole of Fig. 6. By using Eq. (12) and Eq. (14), the resulting offsets are $x_{l}^{0}=311.3 \pm 2.8 \mu \mathrm{m}$ and $y_{l}^{0}=288.3 \pm 2.3 \mu \mathrm{m}$. The data shown here corresponds to the case $l=10$. uncertainty. In particular one can check that a $\pi / 2$ rotation, that is $x \Rightarrow y$ and $y \Rightarrow-x$ which implies $\mathcal{D}_{x} \Rightarrow \mathcal{D}_{y}$, $\mathcal{D}_{y} \Rightarrow-\mathcal{D}_{x}, \mathcal{D}_{x h} \Rightarrow \mathcal{D}_{y h}, \mathcal{D}_{y h} \Rightarrow-\mathcal{D}_{x h}, \mathcal{D}_{x v} \Rightarrow \mathcal{D}_{y v}$ and $\mathcal{D}_{y v} \Rightarrow-\mathcal{D}_{x v}$, leaves Eq. (12) unchanged.

A second example of linear fit to infer the coefficients $\mathcal{M}$ and, thus, the offsets of a dc skew quadrupole next to the BPM $l=10$ is shown in Fig. 6 .

A last example of analysis, this time performed with an ac modulation of the same skew quadrupole of Fig. 6 is shown in Fig. 7. The horizontal offsets inferred with the dc and ac excitation are $330.7 \pm 0.5 \mu \mathrm{m}$ and $311.3 \pm 2.8 \mu \mathrm{m}$, respectively. The vertical offsets are also discordant $265.3 \pm 7.0 \mu \mathrm{m}$ and $288.3 \pm 2.3 \mu \mathrm{m}$. In Sec. V it will be shown how a systematic discrepancy between the results from a dc and an ac skew quadrupole variation affects all magnets at ALBA.

\section{OPTIMIZING THE EXPERIMENTAL SETUP}

In practice, the experimental set-up has a certain parameter settings that can influence the precision of our analysis. For this reason, we provide next a study of these parameters, like the waveform amplitude and frequency or the BPM buffer length. The OCM waveform is defined by a series of discrete current set points separated by $80 \mu$ s and pre-loaded in the magnet power supply. The waveform is repeated continuously so that the current in the corrector coils emulates a sinusoidal profile. However, if the requested rate of change in the current is too large, the output current may not be able to follow the desired curve. For each waveform frequency there is thus an upper limit on the achievable effective sine amplitude. Above that maximum, the effective amplitude will no longer increase and the output current will exhibit spikes and discontinuities on top of the pure sinusoidal signal, introducing harmonics at higher frequencies.

Figure 8 shows the maximum orbit distortion at the 120 ALBA BPMs as a function of the OCM waveform amplitude and frequency. As in the standard BBA, a maximum orbit distortion of around $\pm 0.5 \mathrm{~mm}$ is created during the FBBA. According to Fig. 8 this is only possible at frequencies lower than $7 \mathrm{~Hz}$. On the other hand, from 0 to $15 \mathrm{~Hz}$ the BPMs noise decreases strongly as the frequency increases-see Fig. 9. An amplitude of $0.5 \mathrm{~A}$ and frequencies of 7 and $6 \mathrm{~Hz}$ for the horizontal and vertical plane, respectively, were found to be a reasonable trade-off for the quadrupole FBBA.

ALBA skew quadrupole and OCM power supplies are identical and a similar approach was followed to determine the skew quadrupole frequency in ac mode. During the dc FBBA of normal and skew quadrupoles, their current is varied by $\pm 2.5 \mathrm{~A}$ (the same change of the standard BBA at ALBA). Following an extrapolation of the data in Fig. 8, in order to use the same current change during the ac skew quadrupole FBBA, its frequency is then limited to $1.6 \mathrm{~Hz}$. 


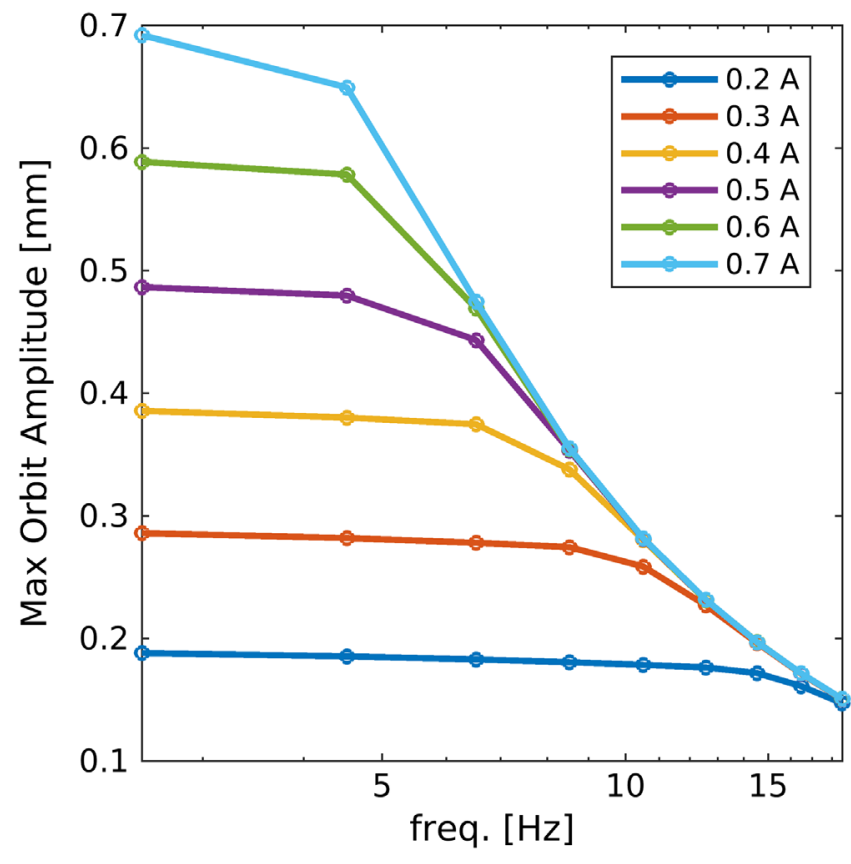

FIG. 8. Maximum orbit distortion over all the 120 BPMs as a function of the waveform amplitude and frequency. A very similar behavior is observed in both planes.

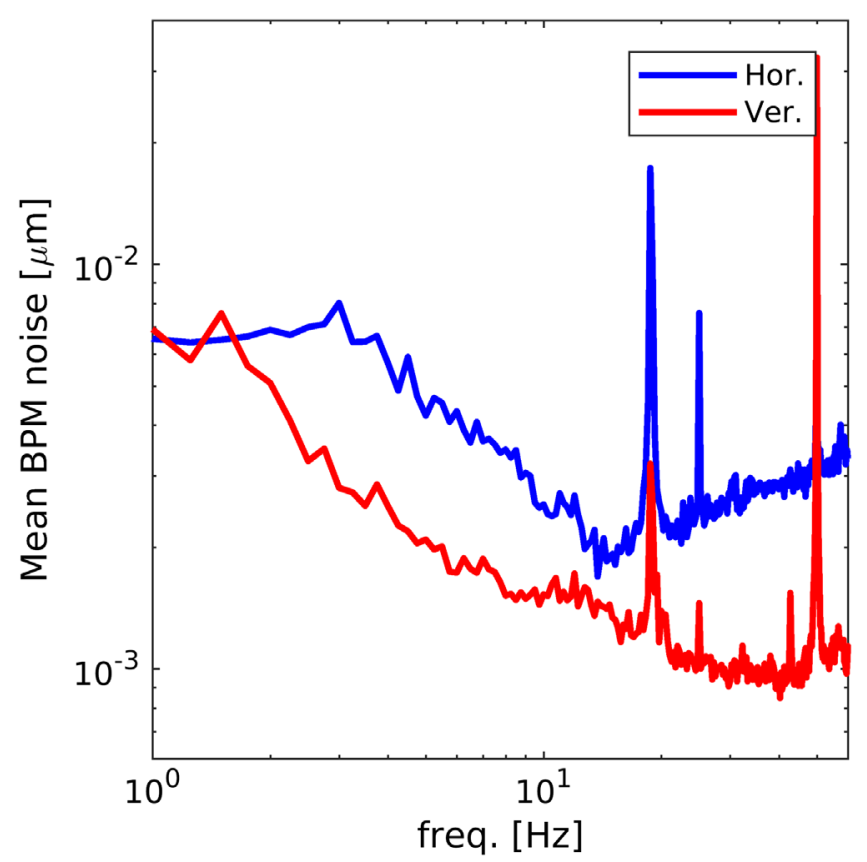

FIG. 9. Example of the 120 BPMs mean spectral noise at $18 \mathrm{~mA}$ (the typical beam current for a BBA measurement at ALBA). The noise decreases rapidly from zero to $15 \mathrm{~Hz}$. It is worthwhile stressing the fact that while the BBA is influenced by the integrated noise spectrum $(1.5 \mu \mathrm{m}$ and $0.8 \mu \mathrm{m} \mathrm{rms}$ in the two planes, including the $50 \mathrm{~Hz}$ peak), the novel FBBA is affected only by the noise at the selected frequencies (which is below $\left.10^{-2} \mu \mathrm{m}\right)$.
The skew quadrupole trim coils are mounted on the sextupole magnets. When the former are powered the magnetic symmetry of the six poles is broken. Magnetic simulations show that the effect of the trim coils is sizable in displacing the magnetic center vertically $(0.1 \mathrm{~mm} / \mathrm{A})$ but also quite linear. For this reason, FBBA of dc quadrupoles are performed both at $-2.5 \mathrm{~A}$ and $+2.5 \mathrm{~A}$. FBBA of ac quadrupole are expected to be less sensitive to this issue because of the continuous and symmetric variation of the trim coil current.

The optimum acquisition time (i.e., the length of the FA buffer to be Fourier analyzed) was determined by evaluating the BPM-to-quad offset errors as a function of the used buffer length. To this end two different sources of error are considered. The first is the uncertainty in evaluating the coefficients $\mathcal{M}_{h}$ and $\mathcal{M}_{v}$ (group error). The second is the error in evaluating the BPM offsets via Eq. (10) (buffer error). Despite the names, both depend on the length of the FA buffer used for the analysis.

For the normal quadrupole FBBA, a test was carried out on 5 BPMs with an acquisition time of $5.5 \mathrm{~s}$ and the resulting offsets were taken as reference. These were then reevaluated after reducing the same FA buffer by steps of $50 \mathrm{~ms}$. Buffer errors between the reference offsets and the ones from the reduced buffer were then computed, and the resulting errors are averaged over the used BPMs subgroup. The group errors are recalculated for every different buffer and averaged for the 5 BPMs. Figure 10 presents an example of such error analysis. For acquisition times of around $1.5 \mathrm{~s}$, both buffer and group errors are below $3 \mu \mathrm{m}$.

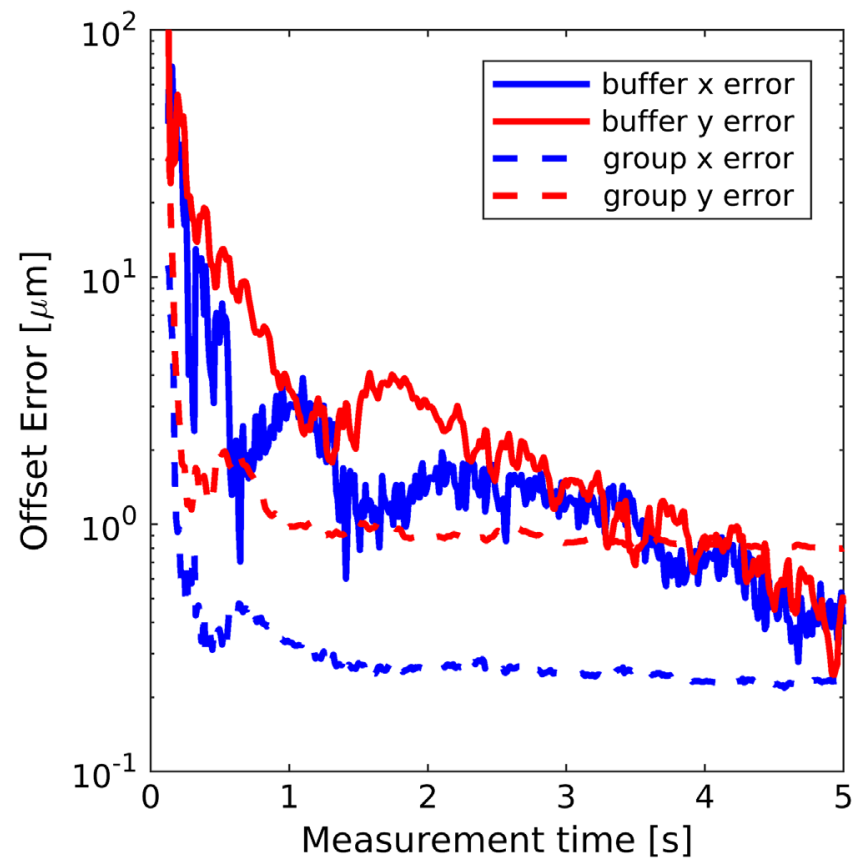

FIG. 10. Measured buffer and group offset errors, averaged over 5 BPMs, as a function of the acquisition time for a (dc) normal quadrupole FBBA. 


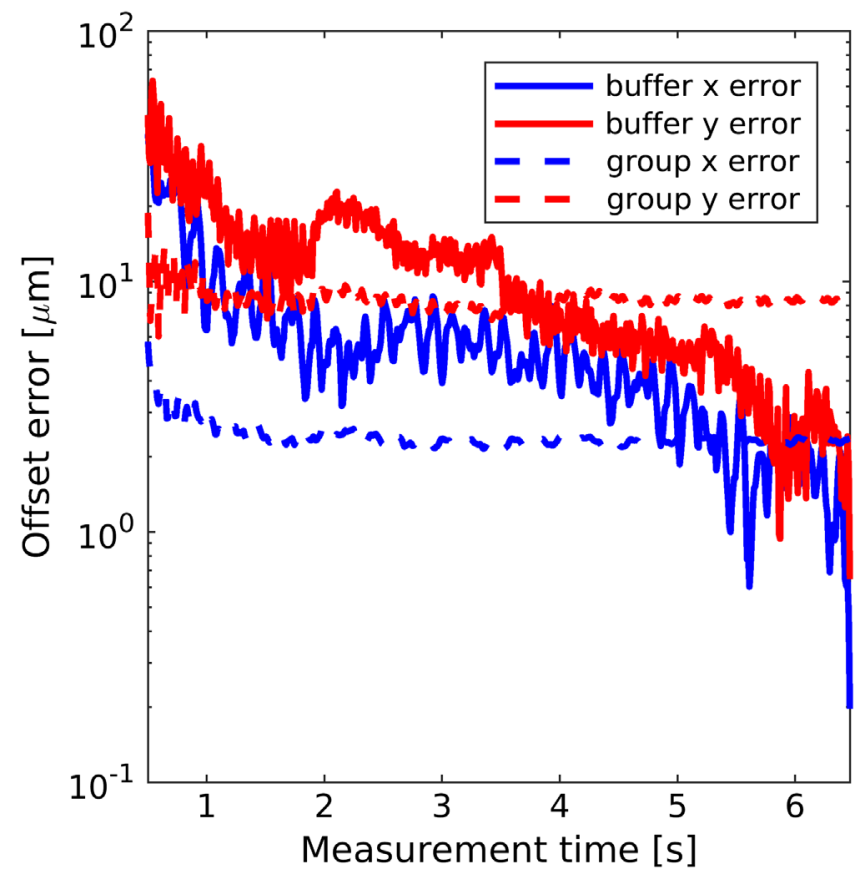

FIG. 11. Measured average of 5 BPM offset errors as a function of the acquisition time for a (dc) skew quadrupole FBBA.

Longer acquisition times would further reduce systematic errors, though at slower pace and at the price of longer numerical calculations. An acquisition time of $1.5 \mathrm{~s}$ was found then to be a acceptable trade-off.

Notice that in Fig. 10 the group error rapidly (above $0.5 \mathrm{~s}$ ) converges to a given value, and it does not decrease

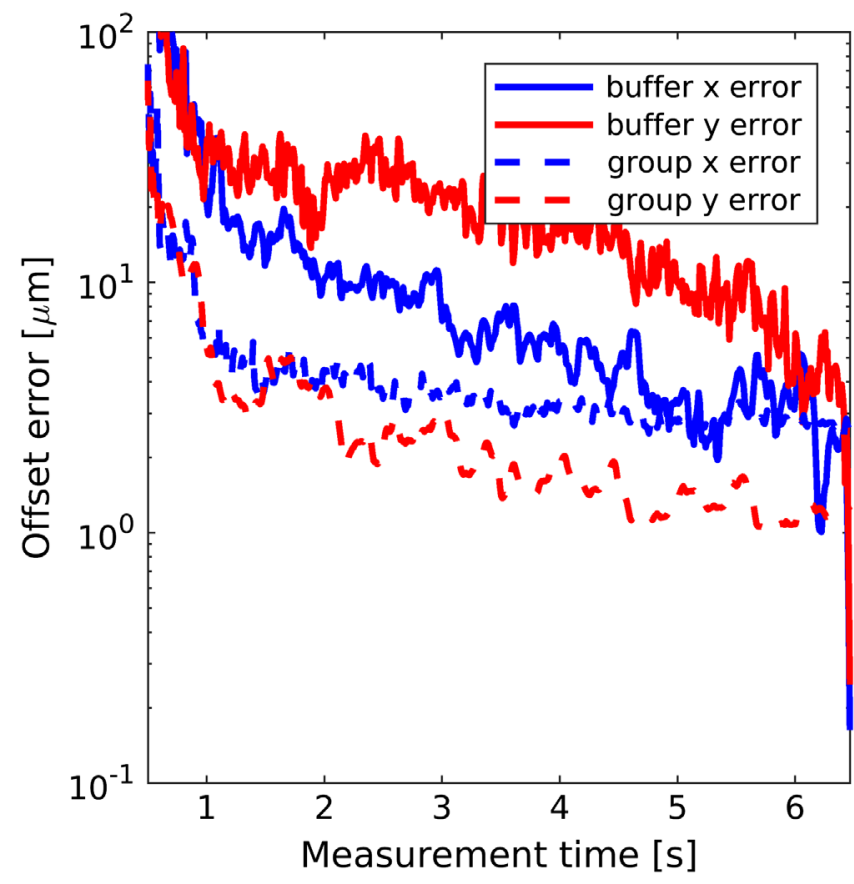

FIG. 12. Measured average of 5 BPM offset errors as a function of the acquisition time for a ac skew quadrupole FBBA.
TABLE I. Summary of the optimized measurement parameters for which the expected buffer error in the evaluation of the offset is around $3 \mu \mathrm{m}$, and the group error of about $1 \mu \mathrm{m}$ for the normal quadrupole case and $10 \mu \mathrm{m}$ in the skew quadrupole case.

OCM horizontal frequency $f_{h}$

$7 \mathrm{~Hz}$

OCM vertical frequency $f_{v}$

$6 \mathrm{~Hz}$

ac skew quadrupole frequency $f_{s}$

$1.6 \mathrm{~Hz}$

rms orbit distortion $(\mathrm{H}, \mathrm{V})$

$0.5 \mathrm{~mm}$

$\Delta \mathrm{I}$ OCM

$0.5 \mathrm{~A}$

$\Delta \mathrm{I}$ normal quadrupole

$2.5 \mathrm{~A}$

$\Delta \mathrm{I}$ skew quadrupole

$2.5 \mathrm{~A}$

Acquisition time (normal quadrupole)

$1.5 \mathrm{~s}$

Acquisition time (skew quadrupole)

with the buffer size. In that case, the group error is limited by a real discrepancy between BPMs in the $\mathcal{M}_{h}$ and $\mathcal{M}_{v}$ fit. The value at which the group error saturates indicates how well our linear model given by Eq. (9) corresponds to the reality.

For the dc skew quadrupole FBBA the same error analysis was carried out, this time increasing the buffer size to $6.5 \mathrm{~s}$. The results are shown in Fig. 11. Buffer and group errors in the two planes are similar already after 2 or $3 \mathrm{~s}$, though at a level much higher than the one observed for normal quadrupoles ( $\sim 10 \mu \mathrm{m}$ compared to less than $3 \mu \mathrm{m})$. This was to be expected, since skew quadrupoles have a much weaker impact on the beam motion (and hence on the BPM signal) compared to normal quadrupoles, where the buffer error reaches the $3 \mu \mathrm{m}$ level around $6 \mathrm{~s}$. The group error also is at the level of $3 \mu \mathrm{m}$ in the horizontal plane.

In the case of the ac skew quadrupole FBBA, the buffer and group errors as a function of the acquisition time are shown in Fig. 12. Again, at $6 \mathrm{~s}$ the buffer error reaches the $3 \mu \mathrm{m}$ level. At $6 \mathrm{~s}$ acquisition time the ac method has similar buffer errors but smaller group errors, specially in the vertical plane.

Table I summarizes the optimized parameters of the experimental setup.

The mechanical alignment of both quadrupole magnets and sextupole magnets during the ALBA storage ring installation is detailed in [13]. According to that, the alignment tolerances are in both cases around $30 \mu \mathrm{m}$. Also, both for quadrupoles and sextupoles, the coils mounted in the iron poles are cooled in parallel, which ensures the same equilibrium temperature and magnetic stated the poles and hence keeps the magnetic center.

\section{ESTIMATION OF THE METHOD ACCURACY}

In the previous section the dependence of the measurement buffer and group errors on the acquisition time has been studied. There are other sources of uncertainty, for instance the beam orbit angle at the quadrupole to be aligned (the lower, the better) and the distance between the magnet and the nearest BPM (again, the lower, the better). 
TABLE II. Standard deviation values for the Gaussian error distribution used to generate the 100 perturbed lattices.

Girder $(\mathrm{H}, \mathrm{V})$ positioning error

Magnet $(\mathrm{H}, \mathrm{V})$ positioning error

Girder and magnet tilt error

Dipole and quadrupole field error

$150 \mu \mathrm{m}$

$25 \mu \mathrm{m}$

$50 \mu \mathrm{rad}$

$0.1 \%$

The orbit angle is difficult to estimate experimentally, though readily accessible in simulations. In this section, we present results from numerical tests aiming at quantifying the expected accuracy induced by these two factors. Even though ac FBBA cannot be performed at ALBA because the quadrupole power supplies do not have ac capabilities, it is next included in the analysis for completeness.

Standard BBA and novel FBBA (both ac and dc) measurements have been simulated on a set of 100 lattice models including realistic magnet and girder random displacements and tilts, magnetic field errors, as well as an orbit distortion corrected at operational levels. The standard deviations of the (Gaussian) error distributions are listed in Table II.

In order to minimize other sources of uncertainty in these simulations, acquisition time and other experimental parameters of Table I have been artificially chosen so to limit their contribution to the overall accuracy to $0.1 \mu \mathrm{m}$. For each one of the 100 lattice models and quadrupoles to be aligned, the most suitable OCM was also chosen: this is to take into account the different modulation of the lattice functions for each model and hence of the modified effectiveness of the steerers.

The accuracy achieved in simulations is summarized in Table III. As figure of merit, the standard deviation of the difference between the expected and the simulated

TABLE III. Top line: quadrupole rms offset inserted into the model and to be inferred. The corresponding BPM offsets are retrieved by the simulated measurements and the standard deviation with respect to the expected value (model, lines 2-6) or from another technique (BBA or dc FFBA, lines 7-10) is computed at all BPMs and averaged over 100 simulated lattices.

\begin{tabular}{llcc}
\hline \hline & & Horizontal & Vertical \\
\hline 1 & Model rms quadrupole offset & $150 \mu \mathrm{m}$ & $150 \mu \mathrm{m}$ \\
& Mean difference between offsets: & & \\
2 & (Normal quad.) BBA vs model & $15 \mu \mathrm{m}$ & $12 \mu \mathrm{m}$ \\
3 & (Normal quad.) dc FBBA vs model & $16 \mu \mathrm{m}$ & $12 \mu \mathrm{m}$ \\
4 & (Normal quad.) ac FBBA vs model & $16 \mu \mathrm{m}$ & $13 \mu \mathrm{m}$ \\
5 & (Skew quad.) dc FBBA vs model & $19 \mu \mathrm{m}$ & $9 \mu \mathrm{m}$ \\
6 & (Skew quad.) ac FBBA vs model & $19 \mu \mathrm{m}$ & $6 \mu \mathrm{m}$ \\
7 & (Normal quad.) dc FBBA vs BBA & $4 \mu \mathrm{m}$ & $2 \mu \mathrm{m}$ \\
8 & (Normal quad.) ac FBBA vs BBA & $4 \mu \mathrm{m}$ & $3 \mu \mathrm{m}$ \\
9 & (Normal quad.) ac FBBA vs dc FBBA & $0 \mu \mathrm{m}$ & $3 \mu \mathrm{m}$ \\
10 & (Skew quad.) ac FBBA vs dc FBBA & $0 \mu \mathrm{m}$ & $5 \mu \mathrm{m}$ \\
\hline \hline
\end{tabular}

measurement BPM offsets is computed first, and averaged over the 100 model lattices then, to generate a single number for each comparison. In general all methods have a similar accuracy (lines 2-6), which is around 10\% of the expected offsets (first line). Differences among the methods are lower: For normal quadrupole offsets the agreement is below $4 \mu \mathrm{m}$ (lines 7-9), while for skew quadrupoles it is $5 \mu \mathrm{m}$ (last line). These error estimates shall be combined with those estimated from the experimental parameters of Sec. III.

\section{FBBA METHOD VALIDATION}

In this section experimental results with the different FBBA methods introduced in Sec. II are presented. In Sec. VA offsets measured via the standard quadrupole $\mathrm{BBA}$ are compared to the ones obtained via the novel FBBA, ignoring betatron coupling in the latter. In Sec. V B, the impact of the corrections included in the FBBA to account for coupling is evaluated on the same data sets. Finally in Sec. V C, outcomes from the skew quadrupole FBBA via dc and ac excitation are examined.

The ALBA storage ring comprises 32 combinedfunction-bending magnets, 112 quadrupoles, 32 skew quadrupoles, 88 OCMs per plane and 120 BPMs. Both FBBA and BBA aim to align the 120 BPMs (or a subgroup in the case of the skew quadrupoles) to their neighboring magnets. Even though each BPM has at least one quadrupole next to it, there are some quadrupole triplets with no BPM in between.

During the measurement, the ALBA storage ring featured a betatron coupling at the level of $\epsilon_{y} / \epsilon_{x} \simeq 0.7 \%$. The experimental parameters of Table I were used.

\section{A. Quadrupole BBA and FBBA (ignoring betatron coupling)}

The novel quadrupole FBBA is first compared to the standard BBA by ignoring betatron coupling in the harmonic analysis of the former, i.e., following the procedure described in Sec. II A. The BPM-to-quad offsets of the 120 BPMs obtained with the two methods are displayed in Fig. 13. The overall agreement is rather good, although at some BPMs discrepancies are well beyond the estimated uncertainty. The standard deviation among all BPMs of the two sets is around $15 \mu \mathrm{m}$ in both planes. The expected buffer error originating from the finite measurement time is approximately $4 \mu \mathrm{m}$ (see Sec. III) and the one stemming from the lattice characteristics an additional $4 \mu \mathrm{m}$ horizontally and $2 \mu \mathrm{m}$ vertically (see Table III). The observed discrepancy is thus more than two times larger than expected. The larger offsets measured around BPM no. 20 are suspected to arise from important mechanical misalignment, though to date this hypothesis could not be verified.

As far as the measurement reproducibility is concerned, both BBA and FBBA of some quadrupoles were repeated 

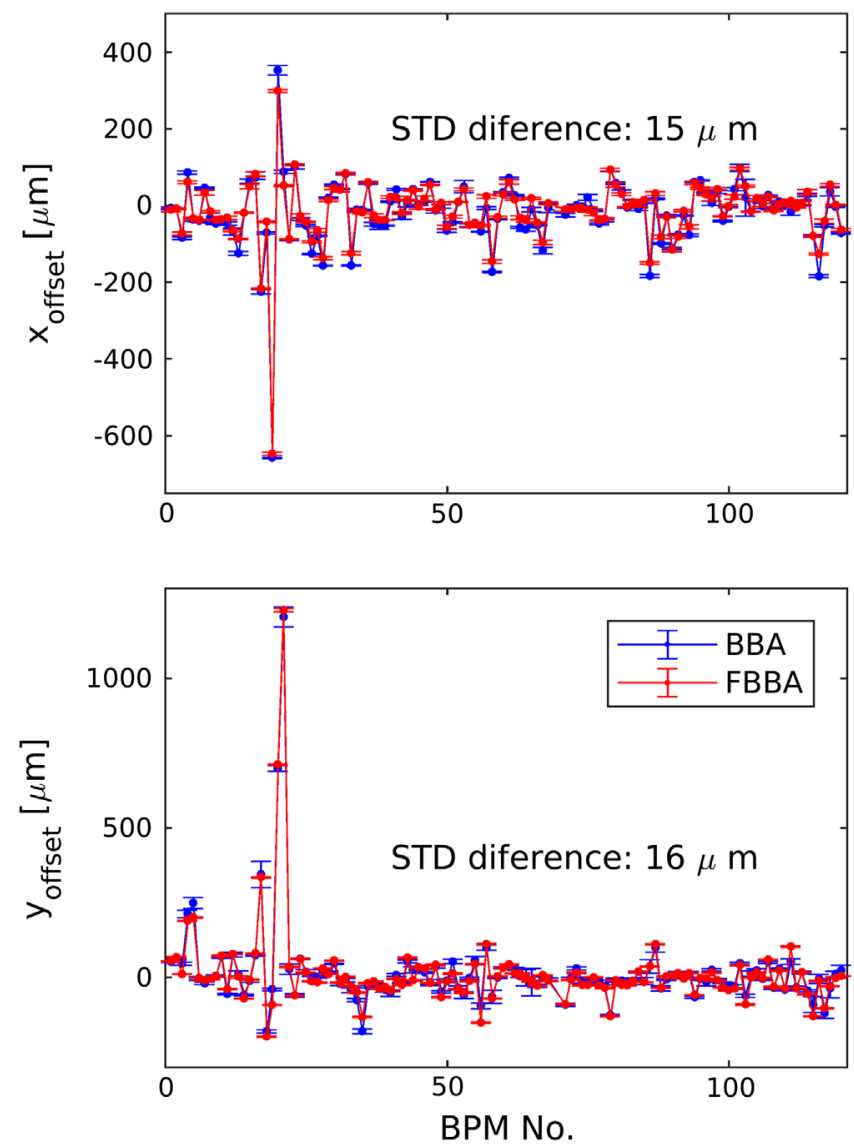

FIG. 13. Standard BBA and FBBA measurements for the 120 ALBA BPMs. The upper plot shows the results for the horizontal plane while the lower plot refers to the vertical plane. The results exciting the OCMs in ac are shown in red while the dc case result is shown in blue.

two consecutive times, and this repeated additional five times after cycling all magnets and waiting ten minutes for thermal stabilization. Results for the horizontal offset at the BPM no. 21 are reported in Fig. 14, showing a global reproducibility within the estimated error bar. Magnetic hysteresis seems also to play no observable role, the curves 1 and 2 in the same plot (without magnet cycling) being compatible with the five values measured after cycling the quadrupoles. Similar observations were made at other BPMs and in the vertical plane. Very similar level of reproducibility were reported in [10].

\section{B. FBBA comparison with beam coupling}

A complete set of quadrupole FBBA is evaluated first by ignoring betatron coupling (i.e., applying the procedure of Sec. II B) and then by including its contribution (i.e., making use of the more general scheme of Sec. IIC). The difference between the 120 BPM offsets inferred from the two approaches is displayed in the two plots of Fig. 15 (left vertical scale). We observe a similar discrepancy in both planes BPM offsets $(6 \mu \mathrm{mrms}$ and $7 \mu \mathrm{m} \mathrm{rms}$ in the

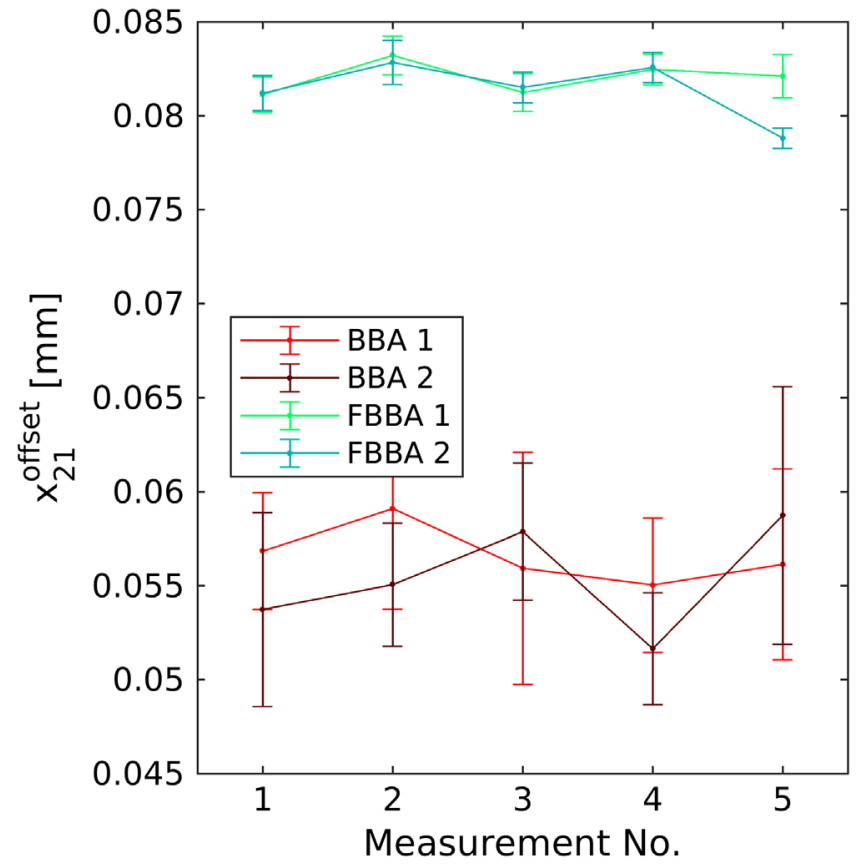

FIG. 14. Reproducibility of both BBA and FBBA for the BPM no. 21 in the horizontal plane over 5 consecutive measurements with (curves 1) and without (curves 2) magnetic cycles.
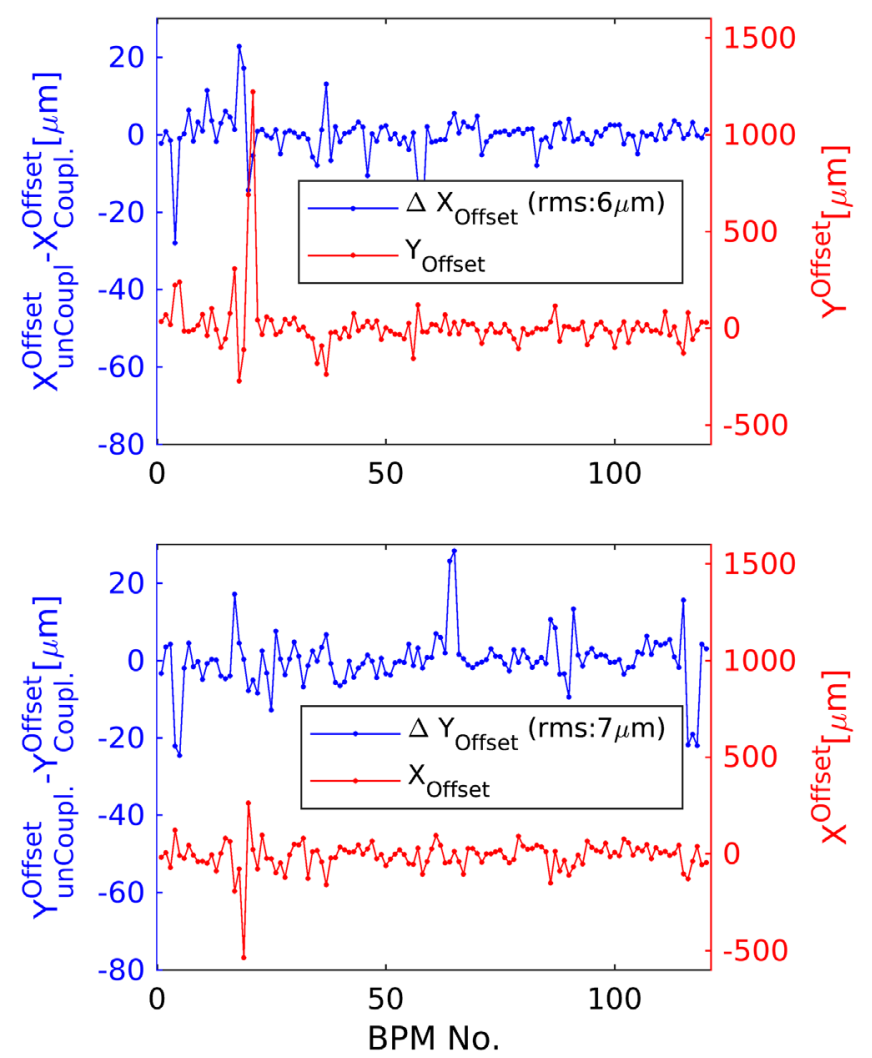

FIG. 15. Difference between the FBBA offsets at the 120 ALBA BPMs when including or excluding coupling effects (blue curves, left axis). In order to attempt to correlate this difference with cross-talk between the two planes, the offsets in the opposite plane are shown in the same plot (red curves, right axis). 
horizontal and vertical plane respectively). Some of the largest differences in the horizontal plane appear to be correlated to the largest vertical offsets (right vertical scale in the top plot of Fig. 15), similarly large horizontal BPM offsets induce large differences between the two approaches in the vertical plane (see bottom plot of the same figure). This correlation, visible mainly around BPM no. 20, is to be expected since the coupling effect is linear with the terms $\mathcal{M}_{h}$ and $\mathcal{M}_{v}$ [see Eq. (10)] so a correlation of the coupling effect and the offset in the opposite plane is expected. Instead, the large discrepancies observed in the vertical plane around BPM No. 60 and BPM No. 115 without any effect in the horizontal plane are not understood.

Also, this comparison provides an estimation of the FBBA and BBA accuracy lower limit for a given amount of coupling ( $0.7 \%$ in our case), BPM rolls and OCM tilts (roughly $1 \%$ in our case): $6 \mu \mathrm{m}$ and $7 \mu \mathrm{m} \mathrm{rms} \mathrm{in} \mathrm{the} \mathrm{two}$ planes, respectively. This is smaller than the discrepancy between standard BBA and FBBA, which is at the level of $15 \mu \mathrm{m}$ rms (see Fig. 13).
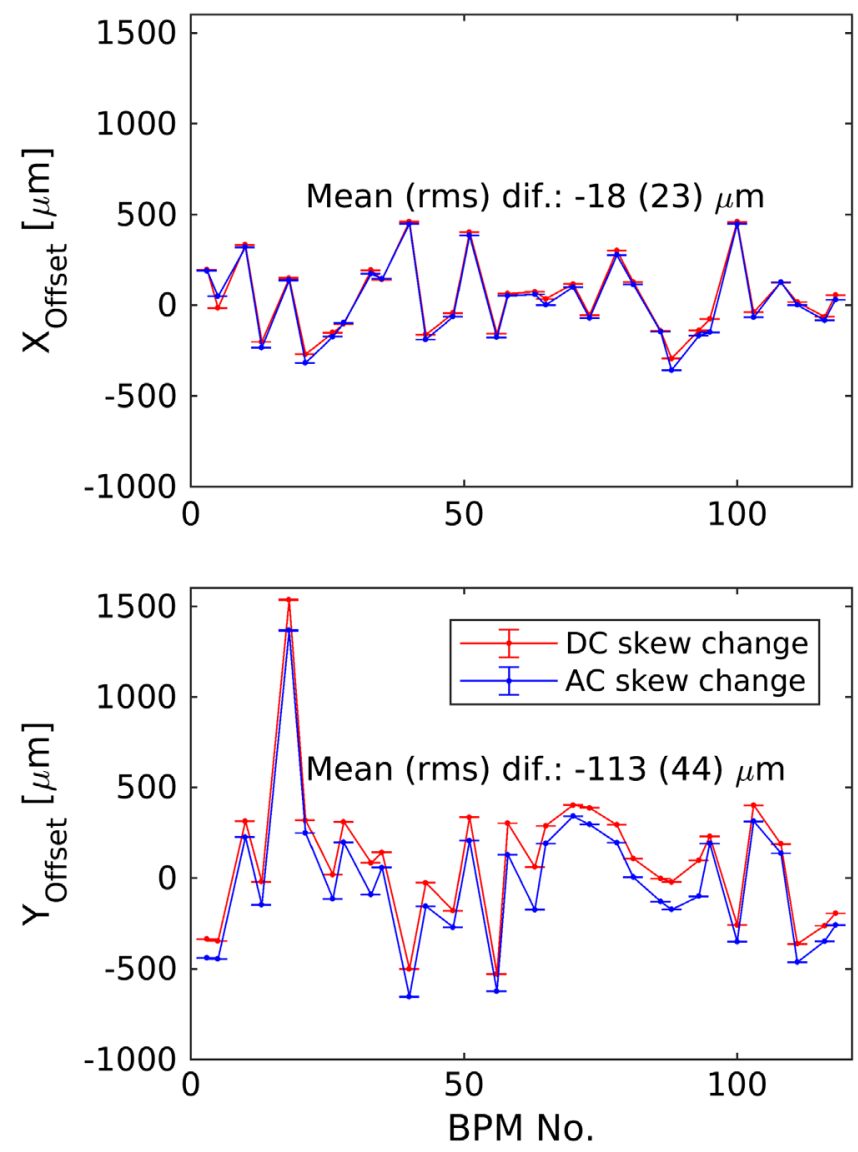

FIG. 16. FBBA measurements at the 32 BPMs nearest to skew quadrupole magnets. The top plot shows the results for the horizontal plane, while the bottom curves refer to the vertical plane. Results obtained via the ac skew quadrupole excitation are shown in blue while those from the dc scan are in red.

\section{Skew quadrupole FBBA comparison}

The FBBA analysis presented in Sec. II C was tested on 32 skew quadrupoles (trim coils on sextupoles), both with $\mathrm{dc}$ and ac excitation. Again, their offset are identified by the one inferred at the nearest BPM. The same optimized experimental parameters of Table I were used. These are expected to provide similar systematic errors (within 4-5 $\mu \mathrm{m}$ ), as shown in Sec. III and Table III. However, the use of an ac excitation reduces the measurement time by a factor 2 .

As shown in Fig. 16, the two methods yield very similar results, especially in the horizontal plane. In the vertical plane, the dc approach results in an almost systematically larger offset (the average difference is $113 \mu \mathrm{m}$ and the rms difference is $44 \mu \mathrm{m}$ ), which corresponds to a factor 20 larger than the one expected from simulations (last row in Table III).

It is worth noticing that in the vertical plane, the dc offsets have an average of $86 \mu \mathrm{m}$ while the ac case the average is quite smaller - $26 \mu \mathrm{m}$. Regarding the method precision, as in the example shown in Sec. II C, the ac case shows smaller error bars (when considering both planes).

As in the case of the normal quadrupole FBBA, repeatability tests at some BPMs (i.e., skew quadrupoles) were performed. The results for BPM no. 21 (the same monitor as in Fig. 14) in the horizontal plane are displayed in Fig. 17. A weak dependence of the BPM offset on the magnetic hysteresis was observed for the dc FBBA during the first two sets of measurements only. That dependency

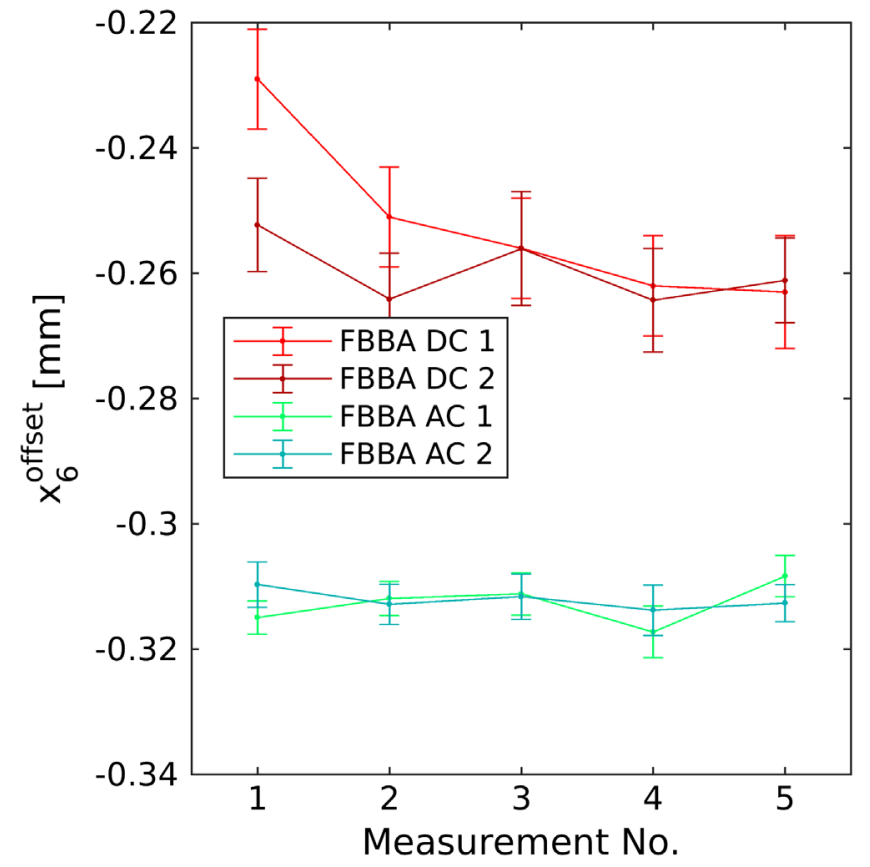

FIG. 17. Reproducibility of both dc and ac skew quadrupole FBBA for the BPM no. 21 (6th skew quadrupole) in the horizontal plane with (curves 1) and without (curves 2) magnetic cycles. 
on the magnetic history in the dc skew quadrupole case was discussed in Sec. III, it should be noticed that although it is a week effect, the magnetic history effect brings the dc measurement closer to the ac measurement. As in Fig. 14 the error bars in Fig. 17 are smaller for the ac case even though they stem from a single measurement.

It is worthwhile noticing how the measured skew quadrupole offsets of Fig. 16 are much larger than those of normal quadrupoles (see Fig. 13), reaching $500 \mu \mathrm{m}$ at several locations, not only in sector 3 (region around BPM no. 20 in Fig. 16) which is suspected to suffer from large mechanical misalignment. According to the mechanical alignment of sextupoles and quadrupoles discussed in Sec. III their difference should be in the range of $30 \mu \mathrm{m}$. The origin of this large discrepancy is unknown and it is one of the crucial issues to tackle in future studies.

\section{CONCLUSIONS}

We have developed a new method (called fast beam based alignment-FBBA) to align the beam through the center of the storage ring quadrupoles by using an ac excitation of the OCMs and the $10 \mathrm{kHz}$ BPM data acquisition - a standard hardware in most synchrotron light sources. The mathematical treatment presented in this paper allows to perform dual plane BBA (horizontal and vertical), includes betatron coupling, BPM roll and OCM tilt effects, and extends this method to both normal and skew quadrupole magnets.

With a careful choice of the experimental setup parameters, the technique speeds up the BBA at ALBA by about a factor 30 (10 min vs 5 hours), and reaches the same level of precision. Furthermore and as shown for the case of the skew quadrupole magnets, the method could still gain a factor two in speed if the quadrupoles could be excited with an ac modulation.

We also performed exhaustive simulations to evaluate the intrinsic accuracy of the method (in the order of $15 \mu \mathrm{m}$ ), and the expected differences between the standard and the FBBA. The experimental results show that both methods agree at the level of the intrinsic accuracy for the normal quadrupole case. Larger and systematic deviations are observed in the vertical offsets of skew quadrupoles depending on their type of variation, $\mathrm{dc}$ or ac. The origin of this discrepancy is still under investigation. Since the dc skew quadrupole measurement is prone to hysteresis effects, we believe it is less accurate that than the ac version. Also the skew quadrupole ac measurement features smaller error bars and is a factor 2 faster.

It is our opinion that the larger scope of the FBBA, namely the inclusion of betatron coupling, BPM roll and OCM tilt and the extension to skew quadrupoles (i.e., to sextupole alignment in most synchrotron light sources), and its huge gain in rapidity allow to consider it as superior with respect to standard beam-based alignment techniques in circular accelerators.

\section{ACKNOWLEDGMENTS}

We would like to thank J. Marcos for the fruitful discussions and his magnetic simulations, and $\mathrm{E}$. Morales and J. Moldes for their contributions to the software and hardware development. We would also like to extend our gratitude to the ALBA Operations and Power Supply Groups for their help.

\section{APPENDIX A: FBBA MATHEMATICAL BACKGROUND}

In this appendix assumptions and mathematics are detailed to derive the final formulas to infer the offset of (either normal or tilted) quadrupoles from observable BPM data from the FA, assuming no knowledge of the ac steerers parameters (strengths and possible tilts). Three hypotheses are needed in order to deduce these formulas: (1) For each quadrupole under study, only one ac steerer per plane is excited at two different frequencies $f_{h}$ and $f_{v}$, respectively. (2) The ac beam orbit distortion at frequencies $f_{h}$ and $f_{v}$ is entirely due to the ac steerers, i.e., any other source of beam motion at those frequencies must be negligible. (3) The amplitude of the ac steerers is also assumed to be invariant during the quadrupole scan.

\section{General formulas}

A more general version of Eq. (1) including betatron coupling reads

$$
\begin{aligned}
& x_{k}\left(t_{n}\right)-x_{k}^{0}=R_{\mathrm{kj}}^{\mathrm{xx}}\left(h_{j}\left(t_{n}\right)-h_{0 j}\right)+R_{\mathrm{kj}}^{\mathrm{xy}}\left(v_{j}\left(t_{n}\right)-v_{0 j}\right), \\
& y_{k}\left(t_{n}\right)-y_{k}^{0}=R_{\mathrm{kj}}^{\mathrm{yx}}\left(h_{j}\left(t_{n}\right)-h_{0 j}\right)+R_{\mathrm{kj}}^{\mathrm{yy}}\left(v_{j}\left(t_{n}\right)-v_{0 j}\right),
\end{aligned}
$$

where $t_{n}$ is the FA sampling time, $R_{\mathrm{kj}}^{\mathrm{xx}}$ and $R_{\mathrm{kj}}^{\mathrm{yy}}$ denote the on-diagonal ORM coefficients, while the off-diagonal ones (generated by betatron coupling) are $R_{\mathrm{kj}}^{\mathrm{xy}}$ and $R_{\mathrm{kj}}^{\mathrm{yx}}$. As for Eq. (1), the index $k$ denotes a generic BPM, whereas the label $l$ will later refer to the monitor closest to the quadrupole under study. The BPM reading corresponding to the centering of the quadrupole will be $\left(x_{l}^{0}, y_{l}^{0}\right)$ obtained with unknown (and not observable) OCM strengths $\left(h_{0 j}, v_{0 j}\right)$. Note that even though 
horizontal and vertical steerers share the same label $j$, they do not necessarily refer to the same magnet: In fact it may be convenient make use of two different OCMs with different horizontal and vertical beta function in order to increase their effectiveness. According to the hypotheses 1, and 2, the beam motion of interest for this study can be Fourier expanded as

$$
\begin{aligned}
& x_{k}\left(t_{n}\right)=\sum_{m=0}^{2} X_{k, m} \cos \left(2 \pi f_{m} t_{n}+\psi_{x_{k}}^{(m)}\right)=\Re\left\{\sum_{m=0}^{2}\left(X_{k, m} e^{i \psi_{x_{k}}^{(m)}}\right) e^{i 2 \pi f_{m} t_{n}}\right\}=\frac{1}{2} \sum_{m=0}^{2}\left(\left\langle x_{k} \mid f_{m}\right\rangle e^{i 2 \pi f_{m} t_{n}}+\text { c.c. }\right), \\
& y_{k}\left(t_{n}\right)=\sum_{m=0}^{2} Y_{k, m} \cos \left(2 \pi f_{m} t_{n}+\psi_{y_{k}}^{(m)}\right)=\Re\left\{\sum_{m=0}^{2}\left(Y_{k, m} e^{i \psi_{y_{k}}^{(m)}}\right) e^{i 2 \pi f_{m} t_{n}}\right\}=\frac{1}{2} \sum_{m=0}^{2}\left(\left\langle y_{k} \mid f_{m}\right\rangle e^{i 2 \pi f_{m} t_{n}}+\text { c.c. }\right),
\end{aligned}
$$

where $\Re$ and c.c. represent the real part and the complex conjugate, respectively, whereas the $\langle z \mid f\rangle$ denotes the (observable) projection of the BPM signal $z$ onto the frequency $f$ (a complex quantity, defined by amplitude and phase of the harmonic at frequency $f$ of the BPM reading, of which more in Appendix B). The above sums run over three indices, $m=0$ (dc mode, $\left.f_{m}=0\right), m=1\left(f_{m}=f_{h}\right)$ and $m=2\left(f_{m}=f_{v}\right)$. The ac steerer functions can be also Fourier expanded. The hypotheses 1 and 2 allow to write them as

$$
\begin{aligned}
& h_{j}\left(t_{n}\right)=\hat{h}_{j} \cos \left(2 \pi f_{h} t_{n}+\psi_{h}\right)=\Re\left\{\left(\hat{h}_{j} e^{i \psi_{h}}\right) e^{i 2 \pi f_{h} t_{n}}\right\}=\frac{1}{2}\left(\left\langle h_{j} \mid f_{h}\right\rangle e^{i 2 \pi f_{h} t_{n}}+\text { c.c. }\right), \\
& v_{j}\left(t_{n}\right)=\hat{v}_{j} \cos \left(2 \pi f_{v} t_{n}+\psi_{v}\right)=\Re\left\{\left(\hat{v}_{j} e^{i \psi_{v}}\right) e^{i 2 \pi f_{v} t_{n}}\right\}=\frac{1}{2}\left(\left\langle v_{j} \mid f_{v}\right\rangle e^{i 2 \pi f_{v} t_{n}}+\text { c.c. }\right) .
\end{aligned}
$$

The amplitudes of the steerer excitation $\hat{h}_{j}$ and $\hat{v}_{j}$ are assumed to be not observable, as the archiving of their currents is either absent or not reliable for a conversion into strengths. The above expressions are valid as long as the OCMs are upright. A steerer tilted by an angle $\omega$ introduces a vertical (horizontal) motion component at frequency $f_{h}\left(f_{v}\right)$, namely

$$
\begin{aligned}
& h_{j}\left(t_{n}\right)=\frac{1}{2}\left(\mathcal{C}_{\omega, j}\left\langle h_{j} \mid f_{h}\right\rangle e^{i 2 \pi f_{h} t_{n}}+\mathcal{S}_{\omega, j}\left\langle v_{j} \mid f_{v}\right\rangle e^{i 2 \pi f_{v} t_{n}}+\text { c.c. }\right), \\
& v_{j}\left(t_{n}\right)=\frac{1}{2}\left(\mathcal{C}_{\omega, j}\left\langle v_{j} \mid f_{v}\right\rangle e^{i 2 \pi f_{v} t_{n}}+\mathcal{S}_{\omega, j}\left\langle h_{j} \mid f_{h}\right\rangle e^{i 2 \pi f_{h} t_{n}}+\text { c.c. }\right) .
\end{aligned}
$$

In the above definitions, $\mathcal{C}_{\omega, j}$ and $\mathcal{S}_{\omega, j}$ denote $\cos \omega_{j}$ and $\sin \omega_{j}$, respectively. By inserting Eqs (A2) and (A4) in Eq. (A1), the latter reads

$$
\begin{aligned}
\frac{1}{2} \sum_{m=0}^{2}\left(\left\langle x_{k} \mid f_{m}\right\rangle e^{i 2 \pi f_{m} t_{n}}+\text { c.c. }\right)-x_{k}^{0}= & \mathcal{C}_{\omega, j}\left[R_{\mathrm{kj}}^{\mathrm{xx}}\left(\frac{1}{2}\left\langle h_{j} \mid f_{h}\right\rangle e^{i 2 \pi f_{h} t_{n}}+\text { c.c. }-h_{0 j}\right)+R_{\mathrm{kj}}^{\mathrm{xy}}\left(\frac{1}{2}\left\langle v_{j} \mid f_{v}\right\rangle e^{i 2 \pi f_{v} t_{n}}+\text { c.c. }-v_{0 j}\right)\right] \\
& +\mathcal{S}_{\omega, j}\left[R_{\mathrm{kj}}^{\mathrm{xx}}\left(\frac{1}{2}\left\langle v_{j} \mid f_{v}\right\rangle e^{i 2 \pi f_{v} t_{n}}+\text { c.c. }-v_{0 j}\right)+R_{\mathrm{kj}}^{\mathrm{xy}}\left(\frac{1}{2}\left\langle h_{j} \mid f_{h}\right\rangle e^{i 2 \pi f_{h} t_{n}}+\text { c.c. }-h_{0 j}\right)\right], \\
\frac{1}{2} \sum_{m=0}^{2}\left(\left\langle y_{k} \mid f_{m}\right\rangle e^{i 2 \pi f_{m} t_{n}}+\text { c.c. }\right)-y_{k}^{0}= & \mathcal{C}_{\omega, j}\left[R_{\mathrm{kj}}^{\mathrm{yx}}\left(\frac{1}{2}\left\langle h_{j} \mid f_{h}\right\rangle e^{i 2 \pi f_{h} t_{n}}+\text { c.c. }-h_{0 j}\right)+R_{\mathrm{kj}}^{\mathrm{yy}}\left(\frac{1}{2}\left\langle v_{j} \mid f_{v}\right\rangle e^{i 2 \pi f_{v} t_{n}}+\text { c.c. }-v_{0 j}\right)\right] \\
& +\mathcal{S}_{\omega, j}\left[R_{\mathrm{kj}}^{\mathrm{yx}}\left(\frac{1}{2}\left\langle v_{j} \mid f_{v}\right\rangle e^{i 2 \pi f_{v} t_{n}}+\text { c.c. }-v_{0 j}\right)+R_{\mathrm{kj}}^{\mathrm{yy}}\left(\frac{1}{2}\left\langle h_{j} \mid f_{h}\right\rangle e^{i 2 \pi f_{h} t_{n}}+\text { c.c. }-h_{0 j}\right)\right] .
\end{aligned}
$$

Note that both sides in the above equations are real numbers, $h_{0 j}$ and $v_{0 j}$ being real quantities too. The above relations must hold for each mode $m=0,1,2$ and at any time $t_{n}$. They can be then split into two separate systems 


$$
\begin{aligned}
\frac{1}{2}\left(\left\langle x_{k} \mid 0\right\rangle+\text { c.c. }\right)-x_{k}^{0} & =-R_{\mathrm{kj}}^{\mathrm{xx}} h_{0 j}-R_{\mathrm{kj}}^{\mathrm{xy}} v_{0 j}, & & (m=0) \\
\frac{1}{2}\left(\left\langle x_{k} \mid f_{h}\right\rangle e^{i 2 \pi f_{h} t_{n}}+\text { c.c. }\right) & =R_{\mathrm{kj}}^{\mathrm{xx}}\left(\frac{1}{2}\left\langle h_{j} \mid f_{h}\right\rangle e^{i 2 \pi f_{h} t_{n}}+\text { c.c. }\right), & & (m=1) \\
\frac{1}{2}\left(\left\langle x_{k} \mid f_{v}\right\rangle e^{i 2 \pi f_{v} t_{n}}+\text { c.c. }\right) & =R_{\mathrm{kj}}^{\mathrm{xy}}\left(\frac{1}{2}\left\langle v_{j} \mid f_{v}\right\rangle e^{i 2 \pi f_{v} t_{n}}+\text { c.c. }\right), & & (m=2), \\
\frac{1}{2}\left(\left\langle y_{k} \mid 0\right\rangle+\text { c.c. }\right)-y_{k}^{0} & =-R_{\mathrm{kj}}^{\mathrm{yx}} h_{0 j}-R_{\mathrm{kj}}^{\mathrm{yy}} v_{0 j}, & & (m=0) \\
\frac{1}{2}\left(\left\langle y_{k} \mid f_{h}\right\rangle e^{i 2 \pi f_{h} t_{n}}+\text { c.c. }\right) & =R_{\mathrm{kj}}^{\mathrm{yx}}\left(\frac{1}{2}\left\langle h_{j} \mid f_{h}\right\rangle e^{i 2 \pi f_{h} t_{n}}+\text { c.c. }\right), & & (m=1) \\
\frac{1}{2}\left(\left\langle y_{k} \mid f_{v}\right\rangle e^{i 2 \pi f_{v} t_{n}}+\text { c.c. }\right) & =R_{\mathrm{kj}}^{\mathrm{yy}}\left(\frac{1}{2}\left\langle v_{j} \mid f_{v}\right\rangle e^{i 2 \pi f_{v} t_{n}}+\text { c.c. }\right), & & (m=2) .
\end{aligned}
$$

In the above expressions the original ORM coefficients have been replaced by effective ones, accounting for the effects of tilted steerers:

$$
\begin{array}{lll}
R_{\mathrm{kj}}^{\mathrm{xx}} \leftrightarrow \mathcal{C}_{\omega, j} R_{\mathrm{kj}}^{\mathrm{xx}}+\mathcal{S}_{\omega, j} R_{\mathrm{kj}}^{\mathrm{xy}} & R_{\mathrm{kj}}^{\mathrm{xy}} \leftrightarrow \mathcal{C}_{\omega, j} R_{\mathrm{kj}}^{\mathrm{xy}}+\mathcal{S}_{\omega, j} R_{\mathrm{kj}}^{\mathrm{xx}} \\
R_{\mathrm{kj}}^{\mathrm{yy}} \leftrightarrow \mathcal{C}_{\omega, j} R_{\mathrm{kj}}^{\mathrm{yy}}+\mathcal{S}_{\omega, j} R_{\mathrm{kj}}^{\mathrm{yx}}, & R_{\mathrm{kj}}^{\mathrm{yx}} \leftrightarrow \mathcal{C}_{\omega, j} R_{\mathrm{kj}}^{\mathrm{yx}}+\mathcal{S}_{\omega, j} R_{\mathrm{kj}}^{\mathrm{yy}} .
\end{array}
$$

Since the ORM coefficients are not observable through this technique and they will not be used in the final formulas, this replacement and thus the steerer tilts do not impact the following derivation and analysis. The dc equations $(m=0)$ can be written as

$$
\begin{aligned}
& \Re\left\{\left\langle x_{k} \mid 0\right\rangle\right\}-x_{k}^{0}=-R_{\mathrm{kj}}^{\mathrm{xx}} h_{0 j}-R_{\mathrm{kj}}^{\mathrm{xy}} v_{0 j} \\
& \Re\left\{\left\langle y_{k} \mid 0\right\rangle\right\}-y_{k}^{0}=-R_{\mathrm{kj}}^{\mathrm{yx}} h_{0 j}-R_{\mathrm{kj}}^{\mathrm{yy}} v_{0 j},
\end{aligned} \quad \Rightarrow \begin{aligned}
& x_{k}^{0}=\Re\left\{\left\langle x_{k} \mid 0\right\rangle\right\}+R_{\mathrm{kj}}^{\mathrm{xx}} h_{0 j}+R_{\mathrm{kj}}^{\mathrm{xy}} v_{0 j} \\
& y_{k}^{0}=\Re\left\{\left\langle y_{k} \mid 0\right\rangle\right\}+R_{\mathrm{kj}}^{\mathrm{yx}} h_{0 j}+R_{\mathrm{kj}}^{\mathrm{yy}} v_{0 j} .
\end{aligned}
$$

The above equations are the starting point for the evaluation of the quadrupole offset $\left(x_{l}^{0}, y_{l}^{0}\right)$. The next step is to manipulate their right-hand side (r.h.s.) in order to make only observable quantities appear. A physics consideration imposes some constraints on the last two equations of both systems in Eqs. (A6)-(A8). Indeed, the ORM coefficients must be real numbers and time-independent. Those equations must then hold simultaneously for both harmonics oscillating at $\pm f_{h}$ and $\pm f_{v}$, resulting in

$$
\begin{array}{ll}
\left\langle x_{k} \mid f_{h}\right\rangle=R_{\mathrm{kj}}^{\mathrm{xx}}\left\langle h_{j} \mid f_{h}\right\rangle & \left\langle y_{k} \mid f_{h}\right\rangle=R_{\mathrm{kj}}^{\mathrm{yx}}\left\langle h_{j} \mid f_{h}\right\rangle \\
\left\langle x_{k} \mid f_{h}\right\rangle^{*}=R_{\mathrm{kj}}^{\mathrm{xx}}\left\langle h_{j} \mid f_{h}\right\rangle^{*} & \left\langle y_{k} \mid f_{h}\right\rangle^{*}=R_{\mathrm{kj}}^{\mathrm{yx}}\left\langle h_{j} \mid f_{h}\right\rangle^{*} \\
\left\langle x_{k} \mid f_{v}\right\rangle=R_{\mathrm{kj}}^{\mathrm{xy}}\left\langle v_{j} \mid f_{v}\right\rangle & \left\langle y_{k} \mid f_{v}\right\rangle=R_{\mathrm{kj}}^{\mathrm{yy}}\left\langle v_{j} \mid f_{v}\right\rangle \\
\left\langle x_{k} \mid f_{v}\right\rangle^{*}=R_{\mathrm{kj}}^{\mathrm{xy}}\left\langle v_{j} \mid f_{v}\right\rangle^{*}, & \left\langle y_{k} \mid f_{v}\right\rangle^{*}=R_{\mathrm{kj}}^{\mathrm{yy}}\left\langle v_{j} \mid f_{v}\right\rangle^{*} .
\end{array}
$$

The (real) ORM coefficients can be written as

$$
R_{\mathrm{kj}}=\left|R_{\mathrm{kj}}\right| e^{i \theta\left(R_{\mathrm{kj}}\right)}, \quad \theta\left(R_{\mathrm{kj}}\right)=\left\{\begin{array}{ll}
0, & \text { if } R_{\mathrm{kj}}>0 \\
\pi, & \text { if } R_{\mathrm{kj}}<0
\end{array} .\right.
$$

The equations in the systems of Eq. (A10) can then be written as

$$
\begin{array}{ll}
\left|\left\langle x_{k} \mid f_{h}\right\rangle\right| e^{ \pm i \psi_{x_{k}}^{(h)}}=\left|R_{\mathrm{kj}}^{\mathrm{xx}}\right|\left|\left\langle h_{j} \mid f_{h}\right\rangle\right| e^{ \pm i\left[\theta\left(R_{\mathrm{kj}}^{\mathrm{xx}}\right)+\psi_{h}\right]} & \left|\left\langle y_{k} \mid f_{h}\right\rangle\right| e^{ \pm i \psi_{y_{k}}^{(h)}}=\left|R_{\mathrm{kj}}^{\mathrm{yx}}\right|\left|\left\langle h_{j} \mid f_{h}\right\rangle\right| e^{ \pm i\left[\theta\left(R_{\mathrm{kj}}^{\mathrm{yx}}\right)+\psi_{h}\right]} \\
\left|\left\langle x_{k} \mid f_{v}\right\rangle\right| e^{ \pm i \psi_{x_{k}}^{(v)}}=\left|R_{\mathrm{kj}}^{\mathrm{xy}}\right|\left|\left\langle v_{j} \mid f_{v}\right\rangle\right| e^{ \pm i\left[\theta\left(R_{\mathrm{kj}}^{\mathrm{xy}}\right)+\psi_{v}\right],} & \left|\left\langle y_{k} \mid f_{v}\right\rangle\right| e^{ \pm i \psi_{y_{k}}^{(v)}}=\left|R_{\mathrm{kj}}^{\mathrm{yy}}\right|\left|\left\langle v_{j} \mid f_{v}\right\rangle\right| e^{ \pm i\left[\theta\left(R_{\mathrm{kj}}^{\mathrm{yy}}\right)+\psi_{v}\right]} .
\end{array}
$$

For the above equations to be simultaneously valid (i.e., irrespective of the sign in the exponential term) BPM phases $\psi_{x, y}^{(h, v)}$ and steerer phases $\psi_{h, v}$ must satisfy the following relations

$$
\begin{array}{ll}
\psi_{x_{k}}^{(h)}-\psi_{h}=\theta\left(R_{\mathrm{kj}}^{\mathrm{xx}}\right) & \psi_{y_{k}}^{(h)}-\psi_{h}=\theta\left(R_{\mathrm{kj}}^{\mathrm{yx}}\right) \\
\psi_{x_{k}}^{(v)}-\psi_{v}=\theta\left(R_{\mathrm{kj}}^{\mathrm{xy}}\right), & \psi_{y_{k}}^{(v)}-\psi_{v}=\theta\left(R_{\mathrm{kj}}^{\mathrm{yy}}\right) .
\end{array}
$$


The sign of any ORM coefficient can be then replaced by the difference between BPM and steerer phase. By combining Eqs (A11)-(A13) the following expressions for the ORM element can be derived

$$
\begin{aligned}
R_{\mathrm{kj}}^{\mathrm{xx}}=\frac{\mathcal{S}\left\{\left\langle x_{k} \mid f_{h}\right\rangle\right\}}{\hat{h}_{j}} & R_{\mathrm{kj}}^{\mathrm{yx}}=\frac{\mathcal{S}\left\{\left\langle y_{k} \mid f_{h}\right\rangle\right\}}{\hat{h}_{j}} \\
R_{\mathrm{kj}}^{\mathrm{xy}}=\frac{\mathcal{S}\left\{\left\langle x_{k} \mid f_{v}\right\rangle\right\}}{\hat{v}_{j}}, & R_{\mathrm{kj}}^{\mathrm{yy}}=\frac{\mathcal{S}\left\{\left\langle y_{k} \mid f_{v}\right\rangle\right\}}{\hat{v}_{j}},
\end{aligned}
$$

where $\hat{h}_{j}=\left|\left\langle h_{j} \mid f_{h}\right\rangle\right|$ and $\hat{v}_{j}=\left|\left\langle v_{j} \mid f_{v}\right\rangle\right|$ are the steerer amplitudes introduced in Eq. (A4) and the signed amplitudes $\mathcal{S}$ are defined as

$$
\begin{array}{ll}
\mathcal{S}\left\{\left\langle x_{k} \mid f_{h}\right\rangle\right\}=\left|\left\langle x_{k} \mid f_{h}\right\rangle\right| \operatorname{sgn}\left\{\cos \left(\psi_{x_{k}}^{(h)}-\psi_{h}\right)\right\} & \mathcal{S}\left\{\left\langle y_{k} \mid f_{h}\right\rangle\right\}=\left|\left\langle y_{k} \mid f_{h}\right\rangle\right| \operatorname{sgn}\left\{\cos \left(\psi_{y_{k}}^{(h)}-\psi_{h}\right)\right\} \\
\mathcal{S}\left\{\left\langle x_{k} \mid f_{v}\right\rangle\right\}=\left|\left\langle x_{k} \mid f_{v}\right\rangle\right| \operatorname{sgn}\left\{\cos \left(\psi_{x_{k}}^{(v)}-\psi_{v}\right)\right\}, & \mathcal{S}\left\{\left\langle y_{k} \mid f_{v}\right\rangle\right\}=\left|\left\langle y_{k} \mid f_{v}\right\rangle\right| \operatorname{sgn}\left\{\cos \left(\psi_{y_{k}}^{(v)}-\psi_{v}\right)\right\} .
\end{array}
$$

Since the argument within the cosine terms are either 0 or $\pi$, the sign of $\mathcal{S}$ is either positive or negative. While BPM amplitudes $\left|\left\langle x, y \mid f_{h, v}\right\rangle\right|$ and phases $\psi_{x, y}^{(h, v)}$ are direct observables from the harmonic analysis of FA data, the steerer phases $\psi_{h, v}$ are not. Nevertheless they can be inferred from the BPM phases by making use of Eqs. (A11) and (A13), since

$$
\begin{aligned}
\psi_{x_{k}}^{(h)}=n_{1} \pi+\psi_{h} \\
\psi_{x_{k}}^{(v)}=n_{2} \pi+\psi_{v} \\
\psi_{y_{k}}^{(h)}=n_{3} \pi+\psi_{h} \\
\psi_{y_{k}}^{(v)}=n_{4} \pi+\psi_{v}
\end{aligned} \quad \Rightarrow \quad \begin{aligned}
& 2 \psi_{x_{k}}^{(h)}=2 n_{1} \pi+2 \psi_{h} \\
& 2 \psi_{x_{k}}^{(v)}=2 n_{2} \pi+2 \psi_{v} \\
& 2 \psi_{y_{k}}^{(h)}=2 n_{3} \pi+2 \psi_{h} \\
& 2 \psi_{y_{k}}^{(v)}=2 n_{4} \pi+2 \psi_{v},
\end{aligned} \quad \Rightarrow \quad \begin{aligned}
& \psi_{h}=\frac{1}{2} \bmod \left(2 \psi_{x_{k}}^{(h)}, 2 \pi\right) \\
& \psi_{v}=\frac{1}{2} \bmod \left(2 \psi_{x_{k}}^{(v)}, 2 \pi\right) \\
& \psi_{h}=\frac{1}{2} \bmod \left(2 \psi_{y_{k}}^{(h)}, 2 \pi\right) \\
& \psi_{v}=\frac{1}{2} \bmod \left(2 \psi_{y_{k}}^{(v)}, 2 \pi\right),
\end{aligned}
$$

where $n_{1,2,3}$ are either 0 or 1 according to the sign of the corresponding ORM coefficient. The last system in the above equation indicates that the steerer phase can be retrieved from the BPM reading in both planes and that it must be the same at all BPMs. This allows to average over the two planes and among all $N$ BPMs, namely

$$
\begin{array}{cc}
\text { with large coupling: } & \text { with ultra - low coupling: } \\
\psi_{h}=\frac{1}{4 N} \sum_{k=1}^{N}\left[\bmod \left(2 \psi_{x_{k}}^{(h)}, 2 \pi\right)+\bmod \left(2 \psi_{y_{k}}^{(h)}, 2 \pi\right)\right] & \psi_{h}=\frac{1}{2 N} \sum_{k=1}^{N} \bmod \left(2 \psi_{x_{k}}^{(h)}, 2 \pi\right) \\
\psi_{v}=\frac{1}{4 N} \sum_{k=1}^{N}\left[\bmod \left(2 \psi_{y_{k}}^{(v)}, 2 \pi\right)+\bmod \left(2 \psi_{x_{k}}^{(v)}, 2 \pi\right)\right], & \psi_{v}=\frac{1}{2 N} \sum_{k=1}^{N} \bmod \left(2 \psi_{y_{k}}^{(v)}, 2 \pi\right),
\end{array}
$$

The steerer phase $\psi_{h, v}$ is hence measurable and so are the signed amplitudes $\mathcal{S}$ of Eq. (A15). The choice of using either the first or the second set of equations above may depend on the amount of coupling in the machine. Indeed the phases $\psi_{y_{k}}^{(h)}$ and $\psi_{x_{k}}^{(v)}$ originate from the BPM signal in the plane orthogonal to the excitation: With ultralow coupling the measurement of that weak signal may be corrupted by noise and other sources, hence reducing accuracy and precision in evaluating the steerer phases.

Once the steerer phases are computed, the first system of Eq. (A16) can be used to asses the quality of the data: The smaller the deviation from $n \pi$ of the difference between BPM and steerer phase, the more robust is the analysis.

With both $\mathcal{S}$ and $\psi_{h, v}$ computed from observable BPM data, we can go back to Eq. (A9), substitute the ORM coefficients with Eq. (A14) and evaluate the latter for the BPM $l$ closest to the quadrupole under study:

$$
\begin{array}{ll}
x_{l}^{0}=\Re\left\{\left\langle x_{l} \mid 0\right\rangle\right\}+\mathcal{S}\left\{\left\langle x_{l} \mid f_{h}\right\rangle\right\} \mathcal{M}_{h}+\mathcal{S}\left\{\left\langle x_{l} \mid f_{v}\right\rangle\right\} \mathcal{M}_{v} & \mathcal{M}_{h}=\frac{h_{0 j}}{\hat{h}_{j}} \\
y_{l}^{0}=\Re\left\{\left\langle y_{l} \mid 0\right\rangle\right\}+\mathcal{S}\left\{\left\langle y_{l} \mid f_{v}\right\rangle\right\} \mathcal{M}_{v}+\mathcal{S}\left\{\left\langle y_{l} \mid f_{h}\right\rangle\right\} \mathcal{M}_{h}, & \mathcal{M}_{v}=\frac{v_{0 j}}{\hat{v}_{j}},
\end{array}
$$


where the coefficients $\mathcal{M}$ are observable and depend on the magnet under study and on the type of its variation (either dc or ac). The formulas in Eq. (A18) are the core of the FBBA. In the next sections, explicit expressions for the coefficients $\mathcal{M}$ will be derived for normal and skew quadrupoles, both with dc and ac excitation.

It is worthwhile noticing that the second terms in the r.h.s. of Eq. (A18) scale with $\mathcal{S}\left\{\left\langle x_{l} \mid f_{h}\right\rangle\right\}$ and $\mathcal{S}\left\{\left\langle y_{l} \mid f_{v}\right\rangle\right\}$, respectively, i.e., with the beam response to the excitation in the corresponding plane: These are the leading terms along with the dc beam responses $\Re\left\{\left\langle x_{l} \mid 0\right\rangle\right\}$ and $\Re\left\{\left\langle y_{l} \mid 0\right\rangle\right\}$. The last terms scale instead with $\mathcal{S}\left\{\left\langle x_{l} \mid f_{v}\right\rangle\right\}$ and $\mathcal{S}\left\{\left\langle y_{l} \mid f_{h}\right\rangle\right\}$, respectively, i.e., with the beam response in the plane orthogonal to the steerer excitation: This in turn scales with (and account for) betatron coupling in the machine and could be ignored in machines operating at ultralow coupling, where $\left|\left\langle x_{l} \mid f_{v}\right\rangle\right| /\left|\left\langle x_{l} \mid f_{h}\right\rangle\right|,\left|\left\langle y_{l} \mid f_{h}\right\rangle\right| /\left|\left\langle y_{l} \mid f_{v}\right\rangle\right| \approx 0.1 \%$. In order to have the same measurement sensitivity in both planes, OCMs shall be chosen so to have $\left|\mathcal{M}_{h}\right| \simeq\left|\mathcal{M}_{v}\right|$.

\section{Coefficients $\mathcal{M}$ for a dc quadrupole}

In this section we derive the coefficients $\mathcal{M}$ for the case of a quadrupole, either normal, skew or partially rotated, whose strength is varied at least one time and the BPM modes measured each time. By doing so, the dc modes of Eq. (A9) during the quadrupole scan read

$$
\begin{array}{ll}
x_{k}^{0}=\Re\left\{\left\langle x_{k 1} \mid 0\right\rangle\right\}+R_{\mathrm{kj} 1}^{\mathrm{xx}} h_{0 j}+R_{\mathrm{kj} 1}^{\mathrm{xy}} v_{0 j} & y_{k}^{0}=\Re\left\{\left\langle y_{k 1} \mid 0\right\rangle\right\}+R_{\mathrm{kj} 1}^{\mathrm{yx}} h_{0 j}+R_{\mathrm{kj} 1}^{\mathrm{yy}} v_{0 j} \\
x_{k}^{0}=\Re\left\{\left\langle x_{k 2} \mid 0\right\rangle\right\}+R_{\mathrm{kj} 2}^{\mathrm{xx}} h_{0 j}+R_{\mathrm{kj} 2}^{\mathrm{xy}} v_{0 j}, & y_{k}^{0}=\Re\left\{\left\langle y_{k 2} \mid 0\right\rangle\right\}+R_{\mathrm{kj} 2}^{\mathrm{yx}} h_{0 j}+R_{\mathrm{kj} 2}^{\mathrm{yy}} v_{0 j},
\end{array}
$$

where the labels 1 and 2 refer to the quadrupole current $I_{0}-\Delta I$ and to $I_{0}+\Delta I$, respectively. While varying the quadrupole strength, neither the BPM readings centering the magnet $\left(x_{k}^{0}, y_{k}^{0}\right)$ nor the dc steerer strengths $\left(\hat{h}_{j}, \hat{v}_{j}\right)$ changes (hypothesis $\mathrm{n}$. $3)$. The difference between the two equations in the above two systems then reads

$$
\begin{aligned}
& \Re\left\{\left\langle x_{k 2} \mid 0\right\rangle\right\}-\Re\left\{\left\langle x_{k 1} \mid 0\right\rangle\right\}=\left(R_{\mathrm{kj} 1}^{\mathrm{xx}}-R_{\mathrm{kj} 2}^{\mathrm{xx}}\right) h_{0 j}+\left(R_{\mathrm{kj} 1}^{\mathrm{xy}}-R_{\mathrm{kj} 2}^{\mathrm{xy}}\right) v_{0 j} \\
& \Re\left\{\left\langle y_{k 2} \mid 0\right\rangle\right\}-\Re\left\{\left\langle y_{k 1} \mid 0\right\rangle\right\}=\left(R_{\mathrm{kj} 1}^{\mathrm{yy}}-R_{\mathrm{kj} 2}^{\mathrm{yy}}\right) v_{0 j}+\left(R_{\mathrm{kj} 1}^{\mathrm{yx}}-R_{\mathrm{kj} 2}^{\mathrm{yx}}\right) h_{0 j} .
\end{aligned}
$$

None of the quantities in the r.h.s. of the above equations is actually observable. Nevertheless, by diving $h_{0 j}$ by $\hat{h}_{j}$ and $v_{0 j}$ by $\hat{v}_{j}$ and applying Eqs. (A14) and (A18), the rhs are rewritten in terms of the coefficients $\mathcal{M}$ and of the observable signed amplitudes $\mathcal{S}$, namely

$$
\begin{aligned}
& \Re\left\{\left\langle x_{k 2} \mid 0\right\rangle\right\}-\Re\left\{\left\langle x_{k 1} \mid 0\right\rangle\right\}=\left(\mathcal{S}\left\{\left\langle x_{k 1} \mid f_{h}\right\rangle\right\}-\mathcal{S}\left\{\left\langle x_{k 2} \mid f_{h}\right\rangle\right\}\right) \mathcal{M}_{h}+\left(\mathcal{S}\left\{\left\langle x_{k 1} \mid f_{v}\right\rangle\right\}-\mathcal{S}\left\{\left\langle x_{k 2} \mid f_{v}\right\rangle\right\}\right) \mathcal{M}_{v} \\
& \Re\left\{\left\langle y_{k 2} \mid 0\right\rangle\right\}-\Re\left\{\left\langle y_{k 1} \mid 0\right\rangle\right\}=\left(\mathcal{S}\left\{\left\langle y_{k 1} \mid f_{h}\right\rangle\right\}-\mathcal{S}\left\{\left\langle y_{k 2} \mid f_{h}\right\rangle\right\}\right) \mathcal{M}_{h}+\left(\mathcal{S}\left\{\left\langle y_{k 1} \mid f_{v}\right\rangle\right\}-\mathcal{S}\left\{\left\langle y_{k 2} \mid f_{v}\right\rangle\right\}\right) \mathcal{M}_{v} .
\end{aligned}
$$

By defining the following observable parameters, all computed from the harmonic analysis of BPM data before and after varying the quadrupole strength

$$
\begin{aligned}
\mathcal{D}_{\mathrm{x}} & =\Re\left\{\left\langle x_{k 2} \mid 0\right\rangle\right\}-\Re\left\{\left\langle x_{k 1} \mid 0\right\rangle\right\} \\
\mathcal{D}_{\mathrm{y}} & =\Re\left\{\left\langle y_{k 2} \mid 0\right\rangle\right\}-\Re\left\{\left\langle y_{k 1} \mid 0\right\rangle\right\} \\
\mathcal{D}_{\mathrm{xh}} & =\mathcal{S}\left\{\left\langle x_{k 2} \mid f_{h}\right\rangle\right\}-\mathcal{S}\left\{\left\langle x_{k 1} \mid f_{h}\right\rangle\right\} \\
\mathcal{D}_{\mathrm{yv}} & =\mathcal{S}\left\{\left\langle y_{k 2} \mid f_{v}\right\rangle\right\}-\mathcal{S}\left\{\left\langle y_{k 1} \mid f_{v}\right\rangle\right\} \\
\mathcal{D}_{\mathrm{xv}} & =\mathcal{S}\left\{\left\langle x_{k 2} \mid f_{h}\right\rangle\right\}-\mathcal{S}\left\{\left\langle x_{k 1} \mid f_{h}\right\rangle\right\} \\
\mathcal{D}_{\mathrm{yh}} & =\mathcal{S}\left\{\left\langle y_{k 2} \mid f_{h}\right\rangle\right\}-\mathcal{S}\left\{\left\langle y_{k 1} \mid f_{h}\right\rangle\right\},
\end{aligned}
$$

Cramer's rule can be used to invert the system of Eq. (A21), yielding 


$$
\begin{gathered}
\mathcal{M}_{h}=-\frac{\mathcal{D}_{x} \mathcal{D}_{\mathrm{yv}}-\mathcal{D}_{\mathrm{xv}} \mathcal{D}_{y}}{\mathcal{D}_{\mathrm{xh}} \mathcal{D}_{\mathrm{yv}}-\mathcal{D}_{\mathrm{xv}} \mathcal{D}_{\mathrm{yh}}}=\frac{\mathcal{Y}_{h k}}{\mathcal{X}_{h k}} \\
\mathcal{M}_{v}=-\frac{\mathcal{D}_{\mathrm{xh}} \mathcal{D}_{y}-\mathcal{D}_{x} \mathcal{D}_{\mathrm{yh}}}{\mathcal{D}_{\mathrm{xh}} \mathcal{D}_{\mathrm{yv}}-\mathcal{D}_{\mathrm{xv}} \mathcal{D}_{\mathrm{yh}}}=\frac{\mathcal{Y}_{v k}}{\mathcal{X}_{v k}} .
\end{gathered}
$$

In principle $\mathcal{M}$ can be evaluated at every BPM $k$ and averaged over all monitors to obtain a more precise value. However, the denominator in Eq. (A23) can be very small, hence risking to spoil the final result. To overcome this difficulty it was found to be more convenient to evaluate the above denominator $\mathcal{X}$ and numerator $\mathcal{Y}$ at all BPMs and to infer the coefficients $\mathcal{M}$ from the slope of their linear fit $\mathcal{Y}_{h k}=\mathcal{M}_{h} \mathcal{X}_{h k}$ and $\mathcal{Y}_{v k}=\mathcal{M}_{v} \mathcal{X}_{v k}$.

\section{Coefficients $\mathcal{M}$ for an ac quadrupole}

In this section we derive the coefficients $\mathcal{M}$ for the case of a quadrupole, either normal, skew or partially rotated, whose strength is varied continuously via an ac (harmonic) excitation during the modulation of the OCMs. This reduces the measurement time by a factor two, since the measurement does not need to be repeated for two (dc) values of the quadrupole strengths. However, this approach requires a more complex mathematical derivation, since the starting point of Eq. (A1) can no longer be used and a more general formalism needs to be deployed. In presence of an ac quadrupole modulation Eq. (A1) reads

$$
\begin{aligned}
& x_{k}\left(t_{n}\right)-x_{k}^{0}=\left(R_{\mathrm{kj}}^{\mathrm{xx}}+\rho_{\mathrm{kj}}^{\mathrm{xx}}\left(t_{n}\right)\right)\left(h_{j}\left(t_{n}\right)-h_{0 j}\right)+\left(R_{\mathrm{kj}}^{\mathrm{xy}}+\rho_{\mathrm{kj}}^{\mathrm{xy}}\left(t_{n}\right)\right)\left(v_{j}\left(t_{n}\right)-v_{0 j}\right), \\
& y_{k}\left(t_{n}\right)-y_{k}^{0}=\left(R_{\mathrm{kj}}^{\mathrm{yy}}+\rho_{\mathrm{kj}}^{\mathrm{yy}}\left(t_{n}\right)\right)\left(v_{j}\left(t_{n}\right)-v_{0 j}\right)+\left(R_{\mathrm{kj}}^{\mathrm{yx}}+\rho_{\mathrm{kj}}^{\mathrm{yx}}\left(t_{n}\right)\right)\left(h_{j}\left(t_{n}\right)-h_{0 j}\right),
\end{aligned}
$$

where $\rho\left(t_{n}\right)=\hat{\rho} \cos \left(2 \pi f_{s} t_{n}+\psi_{s}\right)$ is the ac variation of the ORM coefficients induced by the quadrupole excitation at (known) frequency $f_{s}$ and where $\rho\left(t_{n}\right)=\hat{\rho} \cos \left(2 \pi f_{s} t_{n}+\psi_{s}\right)$ is the ac variation of the ORM coefficients induced by the quadrupole excitation at (known) frequency $f_{s}$ and phase $\psi_{s}$ (unknown a priori but measurable from BPM data as shown later). As for the dc case, both on-diagonal ORM blocks $\left(\rho^{\mathrm{xx}}, \rho^{\mathrm{yy}}\right)$ and off-diagonal $\left(\rho^{\mathrm{xy}}, \rho^{\mathrm{xy}}\right)$ are let varying in order to account for any possible quadrupole rotation. The terms that do not include $\rho\left(t_{n}\right)$ in the above r.h.s. are the same of the dc case studied so far and excite three modes: $m=0\left(f_{m}=0\right), m=1\left(f_{m}=f_{h}\right)$ and $m=2\left(f_{m}=f_{v}\right)$. The new ac terms generate five additional modes in the recorded orbit. The horizontal ac quadrupole terms can indeed be rewritten as

$$
\begin{aligned}
\hat{\rho}_{\mathrm{kj}}^{\mathrm{xx}} \cos \left(2 \pi f_{s} t_{n}+\psi_{s}\right)\left(h_{j}\left(t_{n}\right)-h_{0 j}\right)= & \Re\left\{\hat{\rho}_{\mathrm{kj}}^{\mathrm{xx}} e^{i\left(m_{k j} \pi+\psi_{s}\right)} e^{i 2 \pi f_{s} t_{n}}\right\}\left[\frac{1}{2}\left(\left\langle h_{j} \mid f_{h}\right\rangle e^{i 2 \pi f_{h} t_{n}}+\text { c.c. }\right)-h_{0 j}\right] \\
= & \frac{1}{4}\left(\left\langle\rho_{\mathrm{kj}}^{\mathrm{xx}} \mid f_{s}\right\rangle e^{i 2 \pi f_{s} t_{n}}+\text { c.c. }\right)\left(\left\langle h_{j} \mid f_{h}\right\rangle e^{i 2 \pi f_{h} t_{n}}-h_{0 j}+\text { c.c. }\right) \\
= & \frac{1}{4}\left\langle\rho_{\mathrm{kj}}^{\mathrm{xx}} \mid f_{s}\right\rangle\left\langle h_{j} \mid f_{h}\right\rangle e^{i 2 \pi\left(f_{h}+f_{s}\right) t_{n}}+\frac{1}{4}\left\langle\rho_{\mathrm{kj}}^{\mathrm{xx}} \mid f_{s}\right\rangle *\left\langle h_{j} \mid f_{h}\right\rangle e^{i 2 \pi\left(f_{h}-f_{s}\right) t_{n}} \\
& -\frac{h_{0 j}}{2}\left\langle\rho_{\mathrm{kj}}^{\mathrm{xx}} \mid f_{s}\right\rangle e^{i 2 \pi f_{s} t_{n}}+\text { c.c. }, \\
\hat{\rho}_{\mathrm{kj}}^{\mathrm{xy}} \cos \left(2 \pi f_{s} t_{n}+\psi_{s}\right)\left(v_{j}\left(t_{n}\right)-v_{0 j}\right)= & \Re\left\{\hat{\rho}_{\mathrm{kj}}^{\mathrm{xy}} e^{i\left(n_{k j} \pi+\psi_{s}\right)} e^{i 2 \pi f_{s} t_{n}}\right\}\left[\frac{1}{2}\left(\left\langle v_{j} \mid f_{v}\right\rangle e^{i 2 \pi f_{v} t_{n}}+\text { c.c. }\right)-v_{0 j}\right] \\
= & \frac{1}{4}\left(\left\langle\rho_{\mathrm{kj}}^{\mathrm{xy}} \mid f_{s}\right\rangle e^{i 2 \pi f_{s} t_{n}}+\text { c.c. }\right)\left(\left\langle v_{j} \mid f_{v}\right\rangle e^{i 2 \pi f_{v} t_{n}}-v_{0 j}+\text { c.c. }\right) \\
= & \frac{1}{4}\left\langle\rho_{\mathrm{kj}}^{\mathrm{xy}} \mid f_{s}\right\rangle\left\langle v_{j} \mid f_{v}\right\rangle e^{i 2 \pi\left(f_{v}+f_{s}\right) t_{n}}+\frac{1}{4}\left\langle\rho_{\mathrm{kj}}^{\mathrm{xy}} \mid f_{s}\right\rangle *\left\langle v_{j} \mid f_{v}\right\rangle e^{i 2 \pi\left(f_{v}-f_{s}\right) t_{n}} \\
& -\frac{v_{0 j}}{2}\left\langle\rho_{\mathrm{kj}}^{\mathrm{xy}} \mid f_{s}\right\rangle e^{i 2 \pi f_{s} t_{n}}+\text { c.c. },
\end{aligned}
$$

where c.c. denotes as usual the complex conjugate, $\left\langle\rho_{\mathrm{kj}}^{\mathrm{xx}} \mid f_{s}\right\rangle=\hat{\rho}_{\mathrm{kj}}^{\mathrm{xx}} e^{i\left(m_{k j} \pi+\psi_{s}\right)},\left\langle\rho_{\mathrm{kj}}^{\mathrm{xy}} \mid f_{s}\right\rangle=\hat{\rho}_{\mathrm{kj}}^{\mathrm{xy}} e^{i\left(n_{k j} \pi+\psi_{s}\right)}$. The amplitude of the ORM modulation $\hat{\rho}_{\mathrm{kj}}$ depends on the maximum ac modulation imparted by the quadrupole and is always positive. In order to account for a change in the sign of the ac ORM coefficients along the ring, which depend on the BPM $k$ and steerer $j$, the integer numbers $m_{k j}$ and $n_{k j}$ are introduced in the corresponding phase terms. The five additional horizontal orbit modes oscillate then at the frequencies $\left(f_{h} \pm f_{s}\right),\left(f_{v} \pm f_{s}\right)$ and $f_{s}$. The ac OCM terms $h_{j}\left(t_{n}\right)$ and $v_{j}\left(t_{n}\right)$ is expanded as in Eq. (A4), whereas the BPM reading $x_{k}\left(t_{n}\right)$ is the same of Eq (A2) with the sum now running 
over eight indices $m=0,1, \ldots, 7$. The first three are the same of Eq. (A6), whereas the additional five stem from Eq. (A25) and read

$$
\begin{aligned}
& \frac{1}{2}\left(\left\langle x_{k} \mid f_{h}+f_{s}\right\rangle e^{i 2 \pi\left(f_{h}+f_{s}\right) t_{n}}+\text { c.c. }\right)=\frac{1}{4}\left\langle\rho_{\mathrm{kj}}^{\mathrm{xx}} \mid f_{s}\right\rangle\left\langle v_{j} \mid f_{h}\right\rangle e^{i 2 \pi\left(f_{h}+f_{s}\right) t_{n}}+\text { c.c. }, \quad(m=3) \\
& \frac{1}{2}\left(\left\langle x_{k} \mid f_{h}-f_{s}\right\rangle e^{i 2 \pi\left(f_{h}-f_{s}\right) t_{n}}+\text { c.c. }\right)=\frac{1}{4}\left\langle\rho_{\mathrm{kj}}^{\mathrm{xx}} \mid f_{s}\right\rangle^{*}\left\langle h_{j} \mid f_{h}\right\rangle e^{i 2 \pi\left(f_{h}-f_{s}\right) t_{n}}+\text { c.c. }, \quad(m=4) \\
& \frac{1}{2}\left(\left\langle x_{k} \mid f_{v}+f_{s}\right\rangle e^{i 2 \pi\left(f_{v}+f_{s}\right) t_{n}}+\text { c.c. }\right)=\frac{1}{4}\left\langle\rho_{\mathrm{kj}}^{\mathrm{xy}} \mid f_{s}\right\rangle\left\langle v_{j} \mid f_{v}\right\rangle e^{i 2 \pi\left(f_{v}+f_{s}\right) t_{n}}+\text { c.c. }, \quad(m=5) \\
& \frac{1}{2}\left(\left\langle x_{k} \mid f_{v}-f_{s}\right\rangle e^{i 2 \pi\left(f_{v}-f_{s}\right) t_{n}}+\text { c.c. }\right)=\frac{1}{4}\left\langle\rho_{\mathrm{kj}}^{\mathrm{xy}} \mid f_{s}\right\rangle^{*}\left\langle v_{j} \mid f_{v}\right\rangle e^{i 2 \pi\left(f_{v}-f_{s}\right) t_{n}}+\text { c.c. }, \quad(m=6) \\
& \frac{1}{2}\left(\left\langle x_{k} \mid f_{s}\right\rangle e^{i 2 \pi f_{s} t_{n}}+\text { c.c. }\right)=-\frac{1}{2}\left(h_{0 j}\left\langle\rho_{\mathrm{kj}}^{\mathrm{xx}} \mid f_{s}\right\rangle+v_{0 j}\left\langle\rho_{\mathrm{kj}}^{\mathrm{xy}} \mid f_{s}\right\rangle\right) e^{i 2 \pi f_{s} t_{n}}+\text { c.c. }, \quad(m=7),
\end{aligned}
$$

Once again, the above relations must hold at any time. This implies that all factors in front of the phasors $e^{i 2 \pi f t_{n}}$ of both hand sides must be equal. The following systems of equations are then obtained

$$
\begin{array}{ll}
\left\langle x_{k} \mid f_{h}+f_{s}\right\rangle=\frac{1}{2}\left\langle\rho_{\mathrm{kj}}^{\mathrm{xx}} \mid f_{s}\right\rangle\left\langle h_{j} \mid f_{h}\right\rangle & \left\langle x_{k} \mid f_{h}+f_{s}\right\rangle^{*}=\frac{1}{2}\left\langle\rho_{\mathrm{kj}}^{\mathrm{xx}} \mid f_{s}\right\rangle^{*}\left\langle h_{j} \mid f_{h}\right\rangle^{*} \\
\left\langle x_{k} \mid f_{h}-f_{s}\right\rangle=\frac{1}{2}\left\langle\rho_{\mathrm{kj}}^{\mathrm{xx}} \mid f_{s}\right\rangle^{*}\left\langle h_{j} \mid f_{h}\right\rangle & \left\langle x_{k} \mid f_{h}-f_{s}\right\rangle^{*}=\frac{1}{2}\left\langle\rho_{\mathrm{kj}}^{\mathrm{xx}} \mid f_{s}\right\rangle\left\langle h_{j} \mid f_{h}\right\rangle^{*} \\
\left\langle x_{k} \mid f_{v}+f_{s}\right\rangle=\frac{1}{2}\left\langle\rho_{\mathrm{kj}}^{\mathrm{xy}} \mid f_{s}\right\rangle\left\langle v_{j} \mid f_{v}\right\rangle & \left\langle x_{k} \mid f_{v}+f_{s}\right\rangle^{*}=\frac{1}{2}\left\langle\rho_{\mathrm{kj}}^{\mathrm{xy}} \mid f_{s}\right\rangle^{*}\left\langle v_{j} \mid f_{v}\right\rangle^{*} \\
\left\langle x_{k} \mid f_{v}-f_{s}\right\rangle=\frac{1}{2}\left\langle\rho_{\mathrm{kj}}^{\mathrm{xy}} \mid f_{s}\right\rangle^{*}\left\langle v_{j} \mid f_{v}\right\rangle & \left\langle x_{k} \mid f_{v}-f_{s}\right\rangle^{*}=\frac{1}{2}\left\langle\rho_{\mathrm{kj}}^{\mathrm{xy}} \mid f_{s}\right\rangle\left\langle v_{j} \mid f_{v}\right\rangle^{*} \\
\left\langle x_{k} \mid f_{s}\right\rangle=-v_{0 j}\left\langle\rho_{\mathrm{kj}}^{\mathrm{xy}} \mid f_{s}\right\rangle-h_{0 j}\left\langle\rho_{\mathrm{kj}}^{\mathrm{xx}} \mid f_{s}\right\rangle, & \left\langle x_{k} \mid f_{s}\right\rangle^{*}=-v_{0 j}\left\langle\rho_{\mathrm{kj}}^{\mathrm{xy}} \mid f_{s}\right\rangle^{*}-h_{0 j}\left\langle\rho_{\mathrm{kj}}^{\mathrm{xx}} \mid f_{s}\right\rangle^{*},
\end{array}
$$

which, after some algebra, can be compacted as

$$
\begin{aligned}
& \left|\left\langle x_{k} \mid f_{h}+f_{s}\right\rangle\right| e^{ \pm i\left(\psi_{x_{k}}^{(h+s)}-\psi_{s}-\psi_{h}\right)}=\frac{1}{2} \hat{\rho}_{\mathrm{kj}}^{\mathrm{xx}} \hat{h}_{j} e^{ \pm i m_{k j} \pi} \\
& \left|\left\langle x_{k} \mid f_{h}-f_{s}\right\rangle\right| e^{ \pm i\left(\psi_{x_{k}}^{(h-s)}+\psi_{s}-\psi_{h}\right)}=\frac{1}{2} \hat{\rho}_{\mathrm{kj}}^{\mathrm{xx}} \hat{h}_{j} e^{\mp i m_{k j} \pi} \\
& \left|\left\langle x_{k} \mid f_{v}+f_{s}\right\rangle\right| e^{ \pm i\left(\psi_{x_{k}}^{(v+s)}-\psi_{s}-\psi_{v}\right)}=\frac{1}{2} \hat{\rho}_{\mathrm{kj}}^{\mathrm{xy}} \hat{v}_{j} e^{ \pm i n_{k j} \pi} \\
& \left|\left\langle x_{k} \mid f_{v}-f_{s}\right\rangle\right| e^{ \pm i\left(\psi_{x_{k}}^{(v-s)}+\psi_{s}-\psi_{v}\right)}=\frac{1}{2} \hat{\rho}_{\mathrm{kj}}^{\mathrm{xy}} \hat{v}_{j} e^{\mp i n_{k j} \pi} \\
& \left|\left\langle x_{k} \mid f_{s}\right\rangle\right| e^{ \pm i\left(\psi_{x_{k}}^{(s)}-\psi_{s}\right)}=-v_{0 j} \hat{\rho}_{\mathrm{kj}}^{\mathrm{xy}} e^{ \pm i n_{k j} \pi}-h_{0 j} \hat{\rho}_{\mathrm{kj}}^{\mathrm{xx}} e^{ \pm i m_{k j} \pi} .
\end{aligned}
$$

$\psi_{x_{k}}^{(h \pm s)}, \psi_{x_{k}}^{(v \pm s)}$ and $\psi_{x_{k}}^{(s)}$ denote the observable phases of the new BPM modes, whereas $\psi_{h}$ and $\psi_{v}$ are the steerer phases measurable from the BPM data via Eq. (A17). The phase of the ac quadrupole modulation $\psi_{s}$ is still unknown, though formulas to infer it from BPM data will be given at the end of this section. Once again, for the above equations to be simultaneously valid (i.e., irrespective of the sign in the exponential term) the following relations between the different phases must hold

$$
\begin{aligned}
\psi_{x_{k}}^{(h+s)}-\psi_{s}-\psi_{h} & =m_{k j} \pi \\
\psi_{x_{k}}^{(h-s)}+\psi_{s}-\psi_{h} & =-m_{k j} \pi \\
\psi_{x_{k}}^{(v+s)}-\psi_{s}-\psi_{v} & =n_{k j} \pi \\
\psi_{x_{k}}^{(v-s)}+\psi_{s}-\psi_{v} & =-n_{k j} \pi \\
\psi_{x_{k}}^{(s)}-\psi_{s} & =o_{k j} \pi,
\end{aligned}
$$


which make both sides in all five equations of the system in Eq. (A27) be real quantities. The (unknown) integer $o_{k j}$ is introduced in the last equation in order to account for the sign of its r.h.s.. The signed amplitude $\mathcal{S}$ can be again introduced as

$$
\begin{aligned}
\mathcal{S}\left\{\left\langle x_{k} \mid f_{h}+f_{s}\right\rangle\right\} & =\left|\left\langle x_{k} \mid f_{h}+f_{s}\right\rangle\right| \operatorname{sgn}\left\{\cos \left(\psi_{x_{k}}^{(h+s)}-\psi_{s}-\psi_{h}\right)\right\} \\
\mathcal{S}\left\{\left\langle x_{k} \mid f_{h}-f_{s}\right\rangle\right\} & =\left|\left\langle x_{k} \mid f_{h}-f_{s}\right\rangle\right| \operatorname{sgn}\left\{\cos \left(\psi_{x_{k}}^{(h-s)}+\psi_{s}-\psi_{h}\right)\right\} \\
\mathcal{S}\left\{\left\langle x_{k} \mid f_{v}+f_{s}\right\rangle\right\} & =\left|\left\langle x_{k} \mid f_{v}+f_{s}\right\rangle\right| \operatorname{sgn}\left\{\cos \left(\psi_{x_{k}}^{(v+s)}-\psi_{s}-\psi_{v}\right)\right\} \\
\mathcal{S}\left\{\left\langle x_{k} \mid f_{v}-f_{s}\right\rangle\right\} & =\left|\left\langle x_{k} \mid f_{v}-f_{s}\right\rangle\right| \operatorname{sgn}\left\{\cos \left(\psi_{x_{k}}^{(v-s)}+\psi_{s}-\psi_{v}\right)\right\} \\
\mathcal{S}\left\{\left\langle x_{k} \mid f_{s}\right\rangle\right\} & =\left|\left\langle x_{k} \mid f_{s}\right\rangle\right| \operatorname{sgn}\left\{\cos \left(\psi_{x_{k}}^{(s)}-\psi_{s}\right)\right\},
\end{aligned}
$$

With the above definition, the system of Eq. (A27) can be rewritten as

$$
\begin{aligned}
\mathcal{S}\left\{\left\langle x_{k} \mid f_{h}+f_{s}\right\rangle\right\} & =\frac{1}{2} \hat{\rho}_{\mathrm{kj}}^{\mathrm{xx}} \hat{h}_{j} e^{i m_{k j} \pi} \\
\mathcal{S}\left\{\left\langle x_{k} \mid f_{h}-f_{s}\right\rangle\right\} & =\frac{1}{2} \hat{\rho}_{\mathrm{kj}}^{\mathrm{xx}} \hat{h}_{j} e^{i m_{k j} \pi} \\
\mathcal{S}\left\{\left\langle x_{k} \mid f_{v}+f_{s}\right\rangle\right\} & =\frac{1}{2} \hat{\rho}_{\mathrm{kj}}^{\mathrm{xy}} \hat{v}_{j} e^{i n_{k j} \pi} \\
\mathcal{S}\left\{\left\langle x_{k} \mid f_{v}-f_{s}\right\rangle\right\} & =\frac{1}{2} \hat{\rho}_{\mathrm{kj}}^{\mathrm{xy}} \hat{v}_{j} e^{i n_{k j} \pi} \\
\mathcal{S}\left\{\left\langle x_{k} \mid f_{s}\right\rangle\right\} & =-v_{0 j} \hat{\rho}_{\mathrm{kj}}^{\mathrm{xy}} e^{i n_{k j} \pi}-h_{0 j} \hat{\rho}_{\mathrm{kj}}^{\mathrm{xx}} e^{i m_{k j} \pi},
\end{aligned}
$$

where we have removed the \pm sings since $e^{i m_{k j} \pi}=e^{-i m_{k j} \pi}$. Notice that the projections at $\left(f_{h} \pm f_{s}\right)$ and $\left(f_{v} \pm f_{s}\right)$ generate the same r.h.s in Eq. (A30), but we use them both in order to improve the overall signal to noise ratio. The sum between the equations in the system of Eq. (A27) reads

$$
\mathcal{S}\left\{\left\langle x_{k} \mid f_{s}\right\rangle\right\}=-\left(\mathcal{S}\left\{\left\langle x_{k} \mid f_{v}+f_{s}\right\rangle\right\}+\mathcal{S}\left\{\left\langle x_{k} \mid f_{v}-f_{s}\right\rangle\right\}\right) \frac{v_{0 j}}{\hat{v}_{j}}-\left(\mathcal{S}\left\{\left\langle x_{k} \mid f_{h}+f_{s}\right\rangle\right\}+\mathcal{S}\left\{\left\langle x_{k} \mid f_{h}-f_{s}\right\rangle\right\}\right) \frac{h_{0 j}}{\hat{h}_{j}} .
$$

An equivalent analysis of the vertical signal of Eq. (A24) leads to

$$
\mathcal{S}\left\{\left\langle y_{k} \mid f_{s}\right\rangle\right\}=-\left(\mathcal{S}\left\{\left\langle y_{k} \mid f_{v}+f_{s}\right\rangle\right\}+\mathcal{S}\left\{\left\langle y_{k} \mid f_{v}-f_{s}\right\rangle\right\}\right) \frac{v_{0 j}}{\hat{v}_{j}}-\left(\mathcal{S}\left\{\left\langle y_{k} \mid f_{h}+f_{s}\right\rangle\right\}+\mathcal{S}\left\{\left\langle y_{k} \mid f_{h}-f_{s}\right\rangle\right\}\right) \frac{h_{0 j}}{\hat{h}_{j}} .
$$

Equations (A31) and (A32) can be cast in a linear system equivalent to the one of the dc case, where the unknown coefficients $\mathcal{M}_{h}=h_{0 j} / \hat{h}_{j}$ and $\mathcal{M}_{v}=v_{0 j} / \hat{v}_{j}$ appear:

$$
\begin{aligned}
-\mathcal{D}_{\mathrm{x}} & =\mathcal{D}_{\mathrm{xh}} \mathcal{M}_{h}+\mathcal{D}_{\mathrm{xv}} \mathcal{M}_{v} \\
-\mathcal{D}_{\mathrm{y}} & =\mathcal{D}_{\mathrm{yh}} \mathcal{M}_{h}+\mathcal{D}_{\mathrm{yv}} \mathcal{M}_{v}
\end{aligned}
$$

where the auxiliary observable terms $\mathcal{D}$ read 


$$
\begin{aligned}
\mathcal{D}_{\mathrm{x}} & =\mathcal{S}\left\{\left\langle x_{k} \mid f_{s}\right\rangle\right\} \\
\mathcal{D}_{\mathrm{y}} & =\mathcal{S}\left\{\left\langle y_{k} \mid f_{s}\right\rangle\right\} \\
\mathcal{D}_{\mathrm{xh}} & =\mathcal{S}\left\{\left\langle x_{k} \mid f_{h}+f_{s}\right\rangle\right\}+\mathcal{S}\left\{\left\langle x_{k} \mid f_{h}-f_{s}\right\rangle\right\} \\
\mathcal{D}_{\mathrm{yv}} & =\mathcal{S}\left\{\left\langle y_{k} \mid f_{v}+f_{s}\right\rangle\right\}+\mathcal{S}\left\{\left\langle y_{k} \mid f_{v}-f_{s}\right\rangle\right\} \\
\mathcal{D}_{\mathrm{xv}} & =\mathcal{S}\left\{\left\langle x_{k} \mid f_{v}+f_{s}\right\rangle\right\}+\mathcal{S}\left\{\left\langle x_{k} \mid f_{v}-f_{s}\right\rangle\right\} \\
\mathcal{D}_{\mathrm{yh}} & =\mathcal{S}\left\{\left\langle y_{k} \mid f_{h}+f_{s}\right\rangle\right\}+\mathcal{S}\left\{\left\langle y_{k} \mid f_{h}-f_{s}\right\rangle\right\} .
\end{aligned}
$$

Once again, Cramer's rule can be used to invert the system of Eq. (A33), yielding

$$
\begin{gathered}
\mathcal{M}_{h}=-\frac{\mathcal{D}_{x} \mathcal{D}_{\mathrm{yv}}-\mathcal{D}_{\mathrm{xv}} \mathcal{D}_{y}}{\mathcal{D}_{\mathrm{xh}} \mathcal{D}_{\mathrm{yv}}-\mathcal{D}_{\mathrm{xv}} \mathcal{D}_{\mathrm{yh}}}=\frac{\mathcal{Y}_{h k}}{\mathcal{X}_{h k}} \\
\mathcal{M}_{v}=-\frac{\mathcal{D}_{\mathrm{xh}} \mathcal{D}_{y}-\mathcal{D}_{x} \mathcal{D}_{\mathrm{yh}}}{\mathcal{D}_{\mathrm{xh}} \mathcal{D}_{\mathrm{yv}}-\mathcal{D}_{\mathrm{xv}} \mathcal{D}_{\mathrm{yh}}}=\frac{\mathcal{Y}_{v k}}{\mathcal{X}_{v k}}
\end{gathered}
$$

Once again, the coefficients $\mathcal{M}$ can be inferred from the slope of their linear fit $\mathcal{Y}_{h k}=\mathcal{M}_{h} \mathcal{X}_{h k}$ and $\mathcal{Y}_{v k}=\mathcal{M}_{v} \mathcal{X}_{v k}$, with $\mathcal{Y}$ and $\mathcal{X}$ evaluated at all BPMs.

In order for the element $\mathcal{M}_{h, v}$ to be observable one step is actually missing. The signed amplitudes $\mathcal{S}$ of Eq. (A29) depend on: (i) measurable amplitude and phase of BPM modes, $\left\{\left\langle z \mid f_{q}\right\rangle\right\}$ and $\psi_{z}^{(q)}$, where $z$ is either $x$ or $y$ and $q$ denotes a generic mode; (ii) the steerer phases $\psi_{h}$ and $\psi_{v}$, which are measurable from the BPM data via Eq. (A17); (iii) the quadrupole phase $\psi_{s}$, which is yet unknown. The latter can be however inferred from the all other phases by means of Eq. (A28), since

$$
\begin{array}{ll}
\psi_{s}=\psi_{x_{k}}^{(h+s)}-\psi_{v}-m_{k j} \pi & \psi_{s}=\psi_{y_{k}}^{(v+s)}-\psi_{h}-p_{k j} \pi \\
\psi_{s}=-\psi_{x_{k}}^{(h-s)}+\psi_{v}+m_{k j} \pi & \psi_{s}=-\psi_{y_{k}}^{(v-s)}+\psi_{h}+p_{k j} \pi \\
\psi_{s}=\psi_{x_{k}}^{(v+s)}-\psi_{v}-n_{k j} \pi & \psi_{s}=\psi_{y_{k}}^{(h+s)}-\psi_{h}-q_{k j} \pi \\
\psi_{s}=-\psi_{x_{k}}^{(v-s)}+\psi_{v}+n_{k j} \pi & \psi_{s}=-\psi_{y_{k}}^{(h-s)}+\psi_{h}+q_{k j} \pi \\
\psi_{s}=\psi_{x_{k}}^{(s)}-o_{k j} \pi, & \psi_{s}=\psi_{y_{k}}^{(s)}-r_{k j} \pi,
\end{array}
$$

where the second system is derived from the same analysis in the vertical plane. None of the integer number $\left(m_{k j}, n_{k j}, o_{k j}\right.$, $p_{k j}, q_{k j}$ and $r_{k j}$ ) needs to be evaluated. indeed, by multiplying all above equations by two and taking only the module of $2 \pi$, the following expressions for $\psi_{s}$ are derived

$$
\begin{array}{ll}
\psi_{s}=\bmod \left(2 \psi_{x_{k}}^{(h+s)}-2 \psi_{h}, 2 \pi\right) / 2 & \psi_{s}=\bmod \left(2 \psi_{y_{k}}^{(v+s)}-2 \psi_{v}, 2 \pi\right) / 2 \\
\psi_{s}=\bmod \left(-2 \psi_{x_{k}}^{(h-s)}+2 \psi_{h}, 2 \pi\right) / 2 & \psi_{s}=\bmod \left(-2 \psi_{y_{k}}^{(v-s)}+2 \psi_{v}, 2 \pi\right) / 2 \\
\psi_{s}=\bmod \left(2 \psi_{x_{k}}^{(v+s)}-2 \psi_{v}, 2 \pi\right) / 2 & \psi_{s}=\bmod \left(2 \psi_{y_{k}}^{(h+s)}-2 \psi_{h}, 2 \pi\right) / 2 \\
\psi_{s}=\bmod \left(-2 \psi_{x_{k}}^{(v-s)}+2 \psi_{v}, 2 \pi\right) / 2 & \psi_{s}=\bmod \left(-2 \psi_{y_{k}}^{(h-s)}+2 \psi_{h}, 2 \pi\right) / 2 \\
\psi_{s}=\bmod \left(2 \psi_{x_{k}}^{(s)}, 2 \pi\right) / 2, & \psi_{s}=\bmod \left(2 \psi_{y_{k}}^{(s)}, 2 \pi\right) / 2,
\end{array}
$$

The above expressions can be eventually properly averaged among themselves and over all BPMs to increase the final accuracy.

\section{APPENDIX B: DISCRETE SIGNAL CORRECTED FOURIER COMPONENT}

This appendix details the procedure (already discussed in Ref. [14]) to extract the Fourier component at the frequency $f_{m}$ of a discrete signal $x\left(t_{n}\right)$, sampled at equally spaced time intervals $t_{n}=n \Delta t$. This harmonic corresponds to the projection of $x$ on $f_{m},\left\langle x \mid f_{m}\right\rangle$, introduced in Eq. (A2). This being a complex number, calculations will be made explicit for the real and imaginary parts separately. These are then used to compute the phase $\psi_{x}^{(m)}$ needed to evaluate the OCM phase $\psi_{h}$ via Eq. (A16). We assume that the signal $x\left(t_{n}\right)$ contains a set of $N_{f}$ harmonics at frequencies $f_{m}$, which are those excited during the FBBA. The signal can thus be expanded as 


$$
\begin{aligned}
x\left(t_{n}\right) & =\sum_{m=0}^{N_{f}} X_{m} \cos \left(2 \pi f_{m} t_{n}+\psi_{x}^{(m)}\right)=\Re\left\{\sum_{m=0}^{N_{f}}\left(X_{m} e^{i \eta \psi_{x}^{(m)}}\right) e^{i 2 \pi f_{m} t_{n}}\right\} \\
& =\Re\left\{\sum_{m=0}^{N_{f}}\left\langle x \mid f_{m}\right\rangle e^{i 2 \pi f_{m} t_{n}}\right\}=\frac{1}{2} \sum_{m=0}^{N_{f}}\left(\left\langle x \mid f_{m}\right\rangle e^{i 2 \pi f_{m} t_{n}}+\left\langle x \mid f_{m}\right\rangle^{*} e^{-i 2 \pi f_{m} t_{n}}\right) .
\end{aligned}
$$

The index $m=0$ is included to account for the dc component too $\left(f_{m}=0\right)$. The first step in computing $\left\langle x \mid f_{m}\right\rangle$ is to evaluate the raw Fourier component at a given frequency $f_{r}$

$$
\begin{aligned}
\left\langle x \mid f_{r}\right\rangle_{\mathrm{raw}} & =\frac{2}{N} \sum_{n=0}^{N-1} x\left(t_{n}\right) e^{-2 \pi i f_{r} t_{n}}=\frac{1}{N} \sum_{n=0}^{N-1} \sum_{m=0}^{N_{f}}\left(\left\langle x \mid f_{m}\right\rangle e^{i 2 \pi f_{m} t_{n}}+\left\langle x \mid f_{m}\right\rangle^{*} e^{-i 2 \pi f_{m} t_{n}}\right) e^{-2 \pi i f_{r} t_{n}} \\
& =\sum_{m=0}^{N_{f}}\left[\left\langle x \mid f_{m}\right\rangle\left(\frac{1}{N} \sum_{n=0}^{N-1} e^{-i 2 \pi\left(f_{r}-f_{m}\right) t_{n}}\right)+\left\langle x \mid f_{m}\right\rangle^{*}\left(\frac{1}{N} \sum_{n=0}^{N-1} e^{-i 2 \pi\left(f_{r}+f_{m}\right) t_{n}}\right)\right] .
\end{aligned}
$$

The signal sampled at $10 \mathrm{kHz}$ implies that $t_{n}=n \Delta t$, with $\Delta t=0.1 \mathrm{~ms} . N$ in the above sums represents the length of FA vector data containing the $x$ signal. The exponential sums within the above parentheses can be written as

$$
\sum_{n=0}^{N-1} e^{-i n \Delta t}=\frac{1-e^{-i N \Delta t}}{1-e^{-i \Delta t}} \Rightarrow \xi_{\mathrm{m}}^{ \pm}=\frac{1}{N} \sum_{n=0}^{N-1} e^{-i 2 \pi\left(f_{r}-f_{m}\right) t_{n}}=\frac{1}{N} \frac{1-e^{-i 2 \pi\left(f_{r} \pm f_{m}\right) N \Delta t}}{1-e^{-i 2 \pi\left(f_{r} \pm f_{m}\right) \Delta t}}
$$

The measured raw projection $\left\langle x \mid f_{r}\right\rangle_{\text {raw }}$ is then a linear combination of all corrected Fourier projections $\left\langle x \mid f_{m}\right\rangle$

$$
\left\langle x \mid f_{r}\right\rangle_{\mathrm{raw}}=\xi_{\mathrm{rm}}^{-}\left\langle x \mid f_{m}\right\rangle+\xi_{\mathrm{rm}}^{+}\left\langle x \mid f_{m}\right\rangle^{*} \Rightarrow\left(\begin{array}{c}
\Re\left\{\left\langle x \mid f_{r}\right\rangle\right\} \\
\Im\left\{\left\langle x \mid f_{r}\right\rangle\right\}
\end{array}\right)_{\mathrm{raw}}=\left(\begin{array}{c}
\Re\left\{\xi_{\mathrm{rm}}^{+}+\xi_{\mathrm{rm}}^{-}\right\} \Im\left\{\xi_{\mathrm{rm}}^{+}-\xi_{\mathrm{rm}}^{-}\right\} \\
\Im\left\{\xi_{\mathrm{rm}}^{+}+\xi_{\mathrm{rm}}^{-}\right\} \Re\left\{\xi_{\mathrm{rm}}^{-}-\xi_{\mathrm{rm}}^{+}\right\}
\end{array}\right)\left(\begin{array}{c}
\Re\left\{\left\langle x \mid f_{m}\right\rangle\right\} \\
\Im\left\{\left\langle x \mid f_{m}\right\rangle\right\}
\end{array}\right) .
$$

The above system is then extended to all $N_{f}$ raw projections [measured via Eq. (B2)], resulting in a square linear system

$$
{\overrightarrow{\langle x \mid f\rangle_{\text {raw }}}}=\overrightarrow{\mathcal{C}\langle x \mid f\rangle} \Rightarrow \overrightarrow{\langle x \mid f\rangle}=\mathcal{C}^{-1} \overrightarrow{\langle x \mid f\rangle}_{\text {raw }}
$$

where both $\overrightarrow{\langle x \mid f\rangle}$ and $\overrightarrow{\langle x \mid f\rangle}$ raw are vectors of $2 N_{f}$ elements and $\mathcal{C}$ is a $2 N_{f} \times 2 N_{f}$ matrix dependent on all $\xi_{\mathrm{rm}}^{ \pm}$terms. $\mathcal{C}$ is close to the identity matrix, with nonzero off-diagonal elements generated by the finite sampling time $\Delta t$ and introducing a cross-talk between all modes. The corrected (i.e., uncoupled) projections $\langle x \mid f\rangle$ can be however inferred from the last (inverted) system of Eq. (B5).

[1] A. Wolski and F. Zimmermann, Closed orbit response to quadrupole strength variation, Lawrence Berkeley National Lab. (LBNL) Report No. LBNL-54360 internal note, 2004.

[2] K. Endo, H. Fukuma, and F. Q. Zhang, Preliminary orbit measurement for beam-based alignment, Proceedings of EPAC1996, Sitges, Spain, edited by S. Myers, A. Paheco, R. Pascual, C. Petit-Jean-Genaz, and J. Poole (1996), p. 1657-1659.

[3] I. Pinayev, Centering of quadrupole family, Nucl. Instrum. Methods Phys. Res., Sect. A 570, 351 (2007).

[4] J. Niedziela, C. Montag, and T. Satogata, Quadrupole beam-based alignment at RHIC, in Proceedings of the 21st Particle Accelerator Conference, Knoxville, TN, 2005 (IEEE, Piscataway, NJ, 2005), p. 3493-3495.
[5] K. R. Schmidt, Misalignments from K-modulation, Proceedings of The Third Workshop on LEP Performance, Chamonix, Franc, edited by J. Poole (1993), p. 139-145.

[6] G.Portmann, D.Robin, and L.Schachinger, Automated beam based alignment of the ALS quadrupoles, Proceedings of EPAC1996, Sitges, Spain, edited by S. Myers, A. Paheco, R. Pascual, C. Petit-Jean-Genaz, and J. Poole (1996), p. 2693-2695.

[7] A. Madur and P. Brunelle, and A. Nadji, Beam based alignment for the storage ring multipoles of synchrotron soleil, in Proceedings of the 10th European Particle Accelerator Conference, Edinburgh, Scotland, 2006 (EPS-AG, Edinburgh, Scotland, 2006), p. 1939-1941. 
[8] M. D. Woodley, J. Nelson, M. Ross, J. Turner, A. Wolski, and K. Kubo, Beam based alignment at the KEK-ATF damping ring, in Proceedings of the 9th European Particle Accelerator Conference, Lucerne, 2004 (EPS-AG, Lucerne, 2004) http://accelconf.web.cern.ch/AccelConf/ e04/, p. 36-38.

[9] M. Munoz, Z. Marti, D. Einfeld, and G. Benedetti, Orbit studies during ALBA commissioning, in Proceedings of the 2nd International Particle Accelerator Conference, San Sebastián, Spain (EPS-AG, Spain, 2011), p. 3020-3022.

[10] P. Tenenbaum and T. O. Raubenheimer, Resolution and systematic limitations in beam-based alignment, Phys. Rev. Accel. Beams 3, 052801 (2000).

[11] M. G. Abbott, G. Rehm, and I. S. Uzun, A new fast data logger and viewer at diamond: The FA archiver,
Proceedings of ICALEPCS2011, Grenoble, France, edited by M. Robichon (2011), pp. 1244-1246.

[12] A. Olmos and U. Iriso, Feedback systems at ALBA, Diagnostics Experts European Light Sources DEELS2015, https://indico.cells.es/indico/event/22/.

[13] S. Gurov et al., ALBA storage ring quadrupoles and sextupoles manufacturing and measurements, in Proceedings of the 23rd Particle Accelerator Conference, Vancouver, Canada, 2009 (IEEE, Piscataway, NJ, 2009), pp. 160-162.

[14] Z. Martí, G. Benedetti, M. Carlà, J. Fraxanet, U. Iriso, J. Moldes, A. Olmos, and R. Petrocelli, Fast orbit response matrix measurements at ALBA, Proceedings of IPAC2017, Copenhagen, Denmark, edited by J. Pranke (2017), p. 365-367. 